\title{
Possible pharmaceutical applications can be developed from naturally occurring phenanthroindolizidine and phenanthroquinolizidine alkaloids
}

\author{
Xian-hui Jia $\cdot$ Huan-xin Zhao $\cdot$ Cheng-lin Du $\cdot$ Wen-zhao Tang $(\mathbb{D} \cdot$ \\ Xiao-jing Wang
}

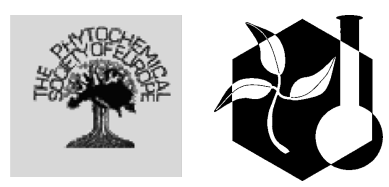

Received: 13 March 2020/Accepted: 14 September 2020/Published online: 25 September 2020

(C) Springer Nature B.V. 2020

\begin{abstract}
Naturally occurring phenanthroindolizidine and phenanthroquinolizidine alkaloids (PIAs and PQAs) are two small groups of herbal metabolites sharing a similar pentacyclic structure with a highly oxygenated phenanthrene moiety fused with a saturated or an unsaturated $N$-heterocycle (indolizidine/quinolizidine moieties). Natural PIAs and PQAs only could be obtained from finite plant families (such as Asclepiadaceae, Lauraceae and Urticaceae families, etc.). Up to date, more than one hundred natural PIAs, while only nine natural PQAs had been described. PIA and PQA analogues have been applied to the development of potent anticancer agents all along because of their excellent cytotoxic activity. However, in the last two decades, other great biological properties, such as anti-inflammatory and antiviral activities were revealed successively by different pharmacological assays. Especially because of their potent antiviral activity against coronavirus (TGEV, SARS CoV and MHV) and tobacco mosaic virus, PIA and PQA analogues have attracted much pharmaceutical attention again, some of them have been used to present interesting targets for total or semi synthesis, and structure-activity relationship (SAR) study for the development of antiviral agents.
\end{abstract}

X. Jia $\cdot$ H. Zhao $\cdot$ C. Du · W. Tang $(\bowtie) \cdot$ X. Wang Institute of Materia Medica, Shandong First Medical University and Shandong Academy of Medical Sciences, Jinan 250062, People's Republic of China

e-mail: twzsd@sina.com
In this review, natural PIA and PQA analogues obtained in the last two decades with their herbal origins, key spectroscopic characteristics for structural identification, biological activity with possible SARs and application prospects were systematically summarized. We hope this paper can stimulate further investigations on PIA and PQA analogues as an important source for potential drug discovery.

Keywords Phenanthroindolizidine and Phenanthroquinolizidine alkaloids · Phytochemistry · Biological properties · Structure-activity relationships $\cdot$ Application prospects

\section{Introduction}

Phenanthroindolizidine and phenanthroquinolizidine alkaloids (PIAs and PQAs) are two small groups of natural bioactive products with similar molecular skeletons, belonging to indolizidine and quinolizidine alkaloid family, respectively. They commonly possess a pentacyclic structure with a phenanthrene moiety fused by a saturated or an unsaturated indolizidine/ quinolizidine group (Fig. 1). Natural PIAs and PQAs only could be obtained from few plant families, such as Asclepiadaceae, Lauraceae and Urticaceae families, etc. (Tables 1 and 2). The first isolation of PIAs was $(R)$-tylophorine (1) from Tylophora indica (Asclepiadaceae family), an herbal medicine used for the 


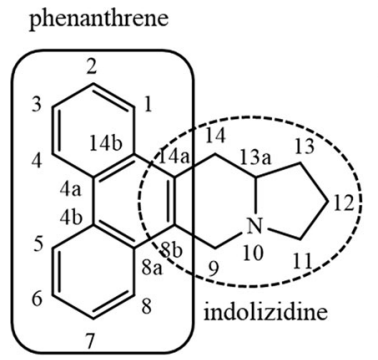

I

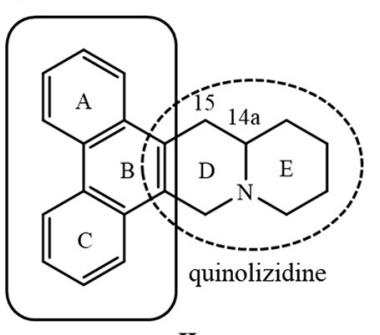

II phenanthrene

Fig. 1 Structure skeletons of PIAs (I) and PQAs (II)

treatment of asthma as well as bronchitis, rheumatism and dysentery in India (Rathnagiriswaran and Venkatachalam 1935). (R)-cryptopleurine (81) was the first isolated PQA analogue from Cryptocarya pleurosperma (Lauraceae family) reported in 1948 (Lande 1948). Up to date, more than one hundred natural PIAs, while only nine natural PQA analogues had been described from herbal origins (Table 3).

Although $(R)$-tylocrebrine (12) was advanced to clinical trials but failed due to its central nervous system (CNS) toxicity, naturally occurring PIAs and PQAs have been applied to the development of potent anticancer agents all along because of their excellent biological activities. Meanwhile, significant effort also has been expended in the total or semi synthesis and structure-activity-relationship (SAR) studies on the anti-cancer activity along with the specific biomolecular targets of these compounds. A series of reviews mainly highlighted important contributions to the PIA and PQA derivatives with synthetic methods and anticancer activities had been summarized successively (Gellert 1982; Li et al. 2001; Chemler 2009; de Fatima et al. 2015), while only Gellert and Li with his co-workers briefly involved those naturally occurring PIAs and PQAs isolated before the year 2000.

In the last two decades, many other new natural PIA and PQA analogues were isolated continuously, and they also have been attracted much attention again because of other biological activities, such as significant anti-inflammatory effect and antiviral activity against coronavirus even in nanomolar level. Furthermore, some natural PIA and PQA analogues, such as $(R)$-antofine (2) and (R)-cryptopleurine (81), displayed great inhibitory effects against plant-pathogenic fungi of Penicillium species, against plant insect of Spodoptera litura and Lipaphis erysimi, and especially against tobacco mosaic virus (TMV). PIA and PQA analogues have represented interesting targets for total or semi synthesis, and SARs study on natural product-based agrochemicals. However, no

Table 1 Herbal origins of natural PIAs

\begin{tabular}{|c|c|c|c|}
\hline No. & Latin name of natural source & Plant family & Name abbreviation \\
\hline 1 & Tylophora indica (Burm. f.) Merr. & Asclepiadaceae & T. indica \\
\hline 2 & Tylophora tanakae Maxim. ex Franch. \& Sav. & & T. tanakae \\
\hline 3 & Tylophora atrofolliculata F.P. Metcalf & & T. atrofolliculata \\
\hline 4 & Tylophora ovata (Lindl.) Hook. ex Steud. & & T. ovata \\
\hline 5 & Vincetoxicum pumilum Decne. & & V. pumilum \\
\hline 6 & Cynanchum vincetoxicum (L.) Pers. & & C. vincetoxicum \\
\hline 7 & Cynanchum komarovii Al. Iljinski & & C. komarovii \\
\hline 8 & Antitoxicum funebre Boiss and Kotschy & & A. funebre \\
\hline 9 & Ficus septica Burm.f. & Moraceae & F. septica \\
\hline 10 & Ficus fistulosa var. tengerensis (Miq.) Kuntze & & F. fistulosa var. tengerensis \\
\hline 11 & Ficus fistulosa Reinw ex. Blume & & F. fistulosa \\
\hline 12 & Ficus hispida L.f. & & F. hispida \\
\hline 13 & Cryptocarya chinensis (Hance) Hemsl. & Lauraceae & C. chinensis \\
\hline 14 & Cryptocarya densiflora Blume & & C. densiflora \\
\hline 15 & Cryptocarya laevigata Blume & & C. laevigata \\
\hline
\end{tabular}

Herbal origins of natural PIAs presented in this Table were described in the literature of isolation only reported after the year 2000 The plant Latin names were presented according to the classification at http://www.theplantlist.org 
Table 2 Herbal origins of natural PQAs

Herbal origins of natural PQAs presented in this Table were all described in the literature of isolation up to date

The plant Latin names were presented according to the classification at http://www. theplantlist.org

\begin{tabular}{llll}
\hline No. & Latin name of natural source & Plant family & Name abbreviation \\
\hline 1 & Cryptocarya pleurosperma White \& Francis & Lauraceae & C. pleurosperma \\
2 & Cryptocarya laevigata Blume & & C. laevigata \\
3 & Boehmeria cylindrica (L.) Sw. & Urticaceae & B. cylindrica \\
4 & Boehmeria siamensis Craib & & B. siamensis \\
5 & Boehmeria pannosa Nakai \& Satake ex Oka & & B. pannosa \\
6 & Boehmeria caudata Sw. & & B. caudata \\
7 & Boehmeria platyphylla Don. & & B. platyphyll \\
8 & Pilea aff. martinii (Lévl.) Hand.-Mazz. & & P. aff. martinii \\
9 & Citsus rheifofia Planch. & Vitaceae & Cheifofia \\
\hline
\end{tabular}

systematic review of various aspects related to these two kinds of analogues has yet been reported at present. In order to offer a comprehensive understanding of PIAs and PQAs, this paper summarized the information from current publications reported after the year 2000, with highlights on their herbal origins, structural variety, key spectroscopic characteristics for structural identification, potent bioactive synthetic derivatives, biological activity with possible SARs and application prospects. We hope the information in this paper can provide an overview on PIAs and PQAs and stimulate further investigation and application on them as pharmaceutical agents.

\section{Occurrence of PIA and PQA analogues}

The first isolation of PIAs was $(R)$-tylophorine (1) from Tylophora indica (Asclepiadaceae family) (Rathnagiriswaran and Venkatachalam 1935), so PIA analogues were also typified as Tylophora alkaloids sometimes. After then, from the other 17 Tylophora species, the trace of PIAs had been discovered by Karnick (Karnick 1975). In the last two decades, from other genera of Asclepiadaceae family, such as Cynanchum, Vincetoxzcum, and Antitoxzcum, a series of PIAs were isolated. Moreover, three species of Cryptocarya genus (Lauraceae family) and four species of Ficus genus (Moraceae family) also could supply natural PIAs.

Natural resources of PQAs were very rare. $(R)$ cryptopleurine (81) was the first isolation of PQA analogue from Cryptocarya pleurosperma (Lande 1948). After then, from the other Cryptocarya species, five species of Boehmeria genus, one species of Pilea (Urticaceae family) and one species of Citsus
(Vitaceae family), another eight PQA analogues were obtained up to date.

Specific herbal origins of isolated PIAs and PQAs were summarized in Tables 1 and 2.

\section{Structural variations of isolated PIAs and PQAs}

Up to date, more than 100 PIAs and nine PQAs have been isolated from natural source. Naturally occurring PIAs and PQAs obtained before the year 2000 had been summarized in a few reviews (Govindachari and Viswanathan 1978; Gellert 1982; Li et al. 2001). This paper mainly mentioned those natural PIA and PQA analogues (including newly isolated and known ones) isolated in the last two decades. Except the different substituents, such as methoxyl or hydroxy group, etc., structural diversity of isolated PIAs and PQAs mainly associated with their skeleton variations of $\mathrm{N}$-oxide (I2), dehydrogenation (I-3) and cycloreversion (I-4 and II-2) (Fig. 2).

For PIA analogues, most of them possessed the basic skeleton I-1. According to the different orientation of the $\mathrm{H}-13 \mathrm{a}$ ( $\alpha$ - or $\beta$-), C-13a possessed $R$ - (1-20, Fig. 3) or $S$ - (21-40, Fig. 4) stereo configuration. Interestingly, recent research indicated the stereochemistry of C-13a might be mainly linked to the methoxy-substitution pattern of the phenanthrene portion. If an oxygen substituent was present at C-2, while C-7 was free, $R$-configuration was observed. However, if C-7 was oxygenated and C-2 was unsubstituted, the $S$-configurated alkaloid was produced instead. If both C-2 and C-7 all carried a methoxy group, a low optical purity was observed (Stoye et al. 2013). Moreover, C-14 of some PIAs with skeleton I-1 could be substituted by a hydroxy group 
Table 3 The distribution of PIAs and PQAs in natural origins

\begin{tabular}{|c|c|c|c|}
\hline Compounds & Name & Origins & References \\
\hline \multicolumn{4}{|l|}{ PIAs* } \\
\hline 1 & $R$-tylophorine & $\begin{array}{l}\text { F. septica } \\
\text { T. tanakae }\end{array}$ & $\begin{array}{l}\text { Damu et al. (2009) } \\
\text { Stærk et al. (2002) } \\
\text { Nakano et al. (2015) }\end{array}$ \\
\hline 2 & $R$-antofine & $\begin{array}{l}\text { V. pumilum } \\
\text { C. komarovii } \\
\text { F. septica } \\
\text { C. vincetoxicum } \\
\text { F. fistulosa } \\
\text { C. densiflora } \\
\text { C. chinensis }\end{array}$ & $\begin{array}{l}\text { Stærk et al. (2005) } \\
\text { An et al. (2001) } \\
\text { Damu et al. (2009) } \\
\text { Stærk et al. (2002) } \\
\text { Subramaniam et al. } \\
\text { (2009) } \\
\text { Othmanan et al. (2017) } \\
\text { Wu et al. (2012) }\end{array}$ \\
\hline 3 & Ficuseptine B & F. septica & Damu et al. (2005) \\
\hline 4 & Ficuseptine C & F. septica & Damu et al. (2005) \\
\hline 5 & Ficuseptine D & F. septica & Damu et al. (2005) \\
\hline 6 & Ficuseptine E & F. septica & Damu et al. (2009) \\
\hline 7 & Ficuseptine F & F. septica & Damu et al. (2009) \\
\hline 8 & Ficuseptine G & F. septica & Damu et al. (2009) \\
\hline 9 & Ficuseptine $\mathrm{H}$ & F. septica & Damu et al. (2009) \\
\hline 10 & Ficuseptine $\mathrm{H}$ & F. septica & Damu et al. (2009) \\
\hline 11 & $R$-isotylocrebrine & F. septica & Damu et al. (2009) \\
\hline 12 & $R$-tylocrebrine & F. septica & Damu et al. (2009) \\
\hline 13 & Tylophoridicine A & T. indica & Dhiman et al. (2013) \\
\hline 14 & $13 \mathrm{a} R$-6-O-desmethylantofine & C. vincetoxicum & Stærk et al. (2002) \\
\hline 15 & 13aR-7-O-desmethyltylophorine & T. tanakae & $\begin{array}{l}\text { Stærk et al. (2002) } \\
\text { Nakano et al. (2015) }\end{array}$ \\
\hline 16 & $13 \mathrm{a} R$-2-hydroxyltylophorinine & T. atrofolliculata & Chen et al. (2016) \\
\hline 17 & Tylophovatine $\mathrm{C}$ & T. ovata & Lee et al. (2011) \\
\hline 18 & O-methyl-tylophorinidine & T. ovata & Lee et al. (2011) \\
\hline 19 & $(13 \mathrm{a} R, 14 S)$-3,6,7-trimethoxy-14-hydrophenanthroindolizidine & T. atrofolliculata & Xiang et al. (2002) \\
\hline 20 & $(13 \mathrm{a} R, 14 S)$-3,7-dimethoxy-6,14-dihydrophenanthroindolizidine & T. atrofolliculata & Xiang et al. (2002) \\
\hline 21 & $S$-tylophorine & $\begin{array}{l}\text { F. septica } \\
\text { T. ovata } \\
\text { T. atrofolliculata }\end{array}$ & $\begin{array}{l}\text { Wu et al. (2002) } \\
\text { Lee et al. (2011) } \\
\text { Chen et al. (2016) }\end{array}$ \\
\hline 22 & 13aS-2,6-didemethyltylophorine & T. atrofolliculata & Chen et al. (2016) \\
\hline 23 & 13aS-6-desmethyltylophorine & T. ovata & Lee et al. (2011) \\
\hline 24 & $(+)$-tylocrebrine & F. septica & Wu et al. (2002) \\
\hline 25 & Deoxytylophorinine (20) & T. atrofolliculata & Chen et al. (2016) \\
\hline 26 & 13aS-3-demethylisotylocrebrine & T. ovata & Lee et al. (2011) \\
\hline 27 & $13 \mathrm{a} S$-isotylocrebrine & $\begin{array}{l}\text { F. septica } \\
\text { T. tanakae }\end{array}$ & $\begin{array}{l}\text { Wu et al. (2002) } \\
\text { Stærk et al. (2002) } \\
\text { Nakano et al. (2015) }\end{array}$ \\
\hline 28 & 2-hydroxyltylophorinidine & T. atrofolliculata & Chen et al. (2016) \\
\hline 29 & 3-O-demethyltylophorinidine & T. atrofolliculata & Chen et al. (2016) \\
\hline
\end{tabular}


Table 3 continued

\begin{tabular}{|c|c|c|c|}
\hline Compounds & Name & Origins & References \\
\hline 30 & Tylophorinidine & $\begin{array}{l}\text { T. indica } \\
\text { T. atrofolliculata }\end{array}$ & $\begin{array}{l}\text { Dhiman et al. (2013) } \\
\text { Xiang et al. (2002) } \\
\text { Chen et al. (2016) }\end{array}$ \\
\hline 31 & $\begin{array}{l}\text { Tylophoridicine E } \\
\text { (13aS,14S)-3,14-dihydroxy- } \\
\text { 6,7dimethoxylphenanthroindolizidine }\end{array}$ & $\begin{array}{l}\text { T. atrofolliculata } \\
\text { T. ovata }\end{array}$ & $\begin{array}{l}\text { Huang et al. (2004) } \\
\text { Chen et al. (2016) } \\
\text { Lee et al. (2011) }\end{array}$ \\
\hline 32 & $\begin{array}{l}\text { O-methyltylophorinidine } \\
\text { (13aS,14S)-3,6,7-trimethoxy-14-hydroxyphenanthroindolizidine }\end{array}$ & T. atrofolliculata & Chen et al. (2016) \\
\hline 33 & $\begin{array}{l}(13 \mathrm{a} S, 14 S)-3,14 \text {-dihydroxy-4,6,7- } \\
\text { trimethoxyphenanthroindolizidine }\end{array}$ & $\begin{array}{l}\text { T. ovata } \\
\text { T. atrofolliculata }\end{array}$ & $\begin{array}{l}\text { Lee et al. (2011) } \\
\text { Xiang et al. (2002) }\end{array}$ \\
\hline 34 & 11-ketotylophorinidine & T. atrofolliculata & Chen et al. (2016) \\
\hline 35 & 11-keto-O-methyltylophorinidine & T. atrofolliculata & Chen et al. (2016) \\
\hline 36 & 6-O- $\beta$-D-glucopyranosyl-tylophorinidine & T. atrofolliculata & Chen et al. (2019a) \\
\hline 37 & Tylophorinine & $\begin{array}{l}\text { T. indica } \\
\text { T. atrofolliculata }\end{array}$ & $\begin{array}{l}\text { Dhiman et al. (2013) } \\
\text { Chen et al. (2016) }\end{array}$ \\
\hline 38 & (13aS,14R)-6,7-dimethoxy-3,14-dihydrophenanthroindolizidine & T. atrofolliculata & Huang et al. (2004) \\
\hline 39 & Hispiloscine & F. hispida & Yap et al. (2015) \\
\hline 40 & Tengechlorenine & $\begin{array}{l}\text { F. fistulosa var. } \\
\text { tengerensis }\end{array}$ & $\begin{array}{l}\text { Al-Khdhairawi et al. } \\
\text { (2017) }\end{array}$ \\
\hline 41 & $10 S, 13 \mathrm{a} R$-antofine $N$-oxide & $\begin{array}{l}V . \text { pumilum } \\
\text { T. indica }\end{array}$ & $\begin{array}{l}\text { Stærk et al. (2005) } \\
\text { Dhiman et al. (2013) }\end{array}$ \\
\hline 42 & $10 S, 13 \mathrm{a} R$-tylocrebrine $N$-oxide & F. septica & $\begin{array}{l}\text { Damu et al. (2005) } \\
\text { Damu et al. (2009) }\end{array}$ \\
\hline 43 & $10 S, 13 \mathrm{a} R$-isotylocrebrine $N$-oxide. & F. septica & $\begin{array}{l}\text { Damu et al. (2005) } \\
\text { Damu et al. (2009) }\end{array}$ \\
\hline 44 & Ficuseptine K & F. septica & Damu et al. (2009) \\
\hline 45 & Ficuseptine L & F. septica & Damu et al. (2009) \\
\hline 46 & Tylophorine $N$-oxide & T. tanakae & Nakano et al. (2015) \\
\hline 47 & (-)-14 $\beta$-hydroxy- $10 \beta, 13 \mathrm{a} \alpha$-antofine $N$-oxide & V. pumilum & Stærk et al. (2005) \\
\hline 48 & 10S-2-hydroxyl-6-demethyltylophorinine $N$-oxide & T. atrofolliculata & Chen et al. (2016) \\
\hline 49 & Tylophorinine $N$-oxide & T. atrofolliculata & Chen et al. (2016) \\
\hline 50 & $14 \beta$-hydroxytylophorine $N$-oxide & T. tanakae & Nakano et al. (2015) \\
\hline 51 & Ficuseptine N & F. septica & Damu et al. (2009) \\
\hline 52 & 3 -demethyl-14 $\alpha$-hydroxyisotylocrebrine $N$-oxide & T. tanakae & Nakano et al. (2015) \\
\hline 53 & $10 R, 13$ a $S$-tylophorine $N$-oxide & $\begin{array}{l}\text { F. septica } \\
\text { T. atrofolliculata }\end{array}$ & $\begin{array}{l}\text { Wu et al. (2002) } \\
\text { Chen et al. (2016) }\end{array}$ \\
\hline 54 & $10 R$-deoxytylophorinine $N$-oxide & T. atrofolliculata & Chen et al. (2016) \\
\hline 55 & $10 R, 14 R$-3-O-demethyltylophorinidine $N$-oxide & T. atrofolliculata & Chen et al. (2016) \\
\hline 56 & 10R-2-hydroxyltylophorinine $N$-oxide & T. atrofolliculata & Chen et al. (2016) \\
\hline 57 & $10 R$-2-methyl-O-methyltylophorinidine $N$-oxide & T. atrofolliculata & Chen et al. (2016) \\
\hline 58 & Ficuseptine $M$ & F. septica & Damu et al. (2009) \\
\hline 59 & Ficuseptine A & F. septica & $\begin{array}{l}\text { Wu et al. (2002) } \\
\text { Damu et al. (2009) }\end{array}$ \\
\hline 60 & $14 \alpha$-hydroxyisotylocrebrine $N$-oxide & F. septica & Wu et al. (2002) \\
\hline
\end{tabular}


Table 3 continued

\begin{tabular}{|c|c|c|c|}
\hline Compounds & Name & Origins & References \\
\hline 61 & $10 S, 13 \mathrm{a} S$-isotylocrebrine $N$-oxide & $\begin{array}{l}\text { F. septica } \\
\text { T. tanakae }\end{array}$ & $\begin{array}{l}\text { Damu et al. (2005) } \\
\text { Nakano et al. (2015) }\end{array}$ \\
\hline 62 & $\begin{array}{l}\text { Tylophoridicine C } \\
(13 \mathrm{a} S, 14 S)-6,14 \text {-dihydroxy-3,7- } \\
\text { dimethoxyphenanthroindolizidine } N \text {-oxide }\end{array}$ & T. atrofolliculata & $\begin{array}{l}\text { Huang et al. (2004) } \\
\text { Chen et al. (2016) }\end{array}$ \\
\hline 63 & 3-demethyl-14 $\alpha$-hydroxyisotylocrebrine $N$-oxide & T. tanakae & Nakano et al. (2015) \\
\hline 64 & $\begin{array}{l}\text { Tylophoridicine F } \\
(13 \mathrm{a} S, 14 R)-3,6,7 \text {-trimethoxy-14-hydrophenanthroindolizidine } \\
N \text {-oxide }\end{array}$ & T. atrofolliculata & Huang et al. (2004) \\
\hline 65 & $10 R, 13 \mathrm{a} R$-tylophorine $N$-oxide & F. septica & Damu et al. (2005) \\
\hline 66 & $10 R, 13 \mathrm{a} R$-tylocrebrine $N$-oxide & F. septica & Damu et al. (2005) \\
\hline 67 & Tylophoridicine D & $\begin{array}{l}\text { T. atrofolliculata } \\
\text { T. indica } \\
\text { F. septica }\end{array}$ & $\begin{array}{l}\text { Chen et al. (2016) } \\
\text { Dhiman et al. (2013) } \\
\text { Kubo et al. (2016) }\end{array}$ \\
\hline 68 & Dehydrotylophorine & F. septica & $\begin{array}{l}\text { Damu et al. (2009) } \\
\text { Kubo et al. (2016) } \\
\text { Wu et al. (2002) }\end{array}$ \\
\hline 69 & Dehydroantofine & $\begin{array}{l}\text { F. septica } \\
\text { C. chinensis }\end{array}$ & $\begin{array}{l}\text { Kubo et al. (2016) } \\
\text { Wu et al. (2012) }\end{array}$ \\
\hline 70 & Ficuseptine I & F. septica & Damu et al. (2009) \\
\hline 71 & $13 \mathrm{a} R$-secoantofine & $\begin{array}{l}\text { C. vincetoxicum } \\
\text { F. fistulosa }\end{array}$ & $\begin{array}{l}\text { Stærk et al. (2002) } \\
\text { Subramaniam et al. } \\
\text { (2009) }\end{array}$ \\
\hline 72 & $13 \mathrm{a} R$-6-O-desmethylsecoantofine & $\begin{array}{l}\text { C. vincetoxicum } \\
\text { C. densiflora }\end{array}$ & $\begin{array}{l}\text { Stærk et al. (2002) } \\
\text { Othman et al. (2017) }\end{array}$ \\
\hline 73 & Fistulopsine A & F. fistulosa & Yap et al. (2016) \\
\hline 74 & Tylophovatine A & T. ovata & Lee et al. (2011) \\
\hline 75 & $(S)-(+)$-hispidine & T. ovata & Lee et al. (2011) \\
\hline 76 & Tylophovatine B & T. ovata & Lee et al. (2011) \\
\hline 77 & $(S)$-(+)-septicine & T. ovata & Lee et al. (2011) \\
\hline 78 & Fistulopsine B & F. fistulosa & Yap et al. (2016) \\
\hline 79 & (-)-desmethylsecoantofine $N$-oxide & C. densiflora & Othmanan et al. (2017) \\
\hline 80 & 4a,b-seco-dehydroantofine & F. septica & Kubo et al. (2016) \\
\hline$P Q A s^{* *}$ & & & \\
\hline 81 & $(R)$-cryptopleurine & $\begin{array}{l}\text { C. pleurosperma } \\
\text { C. laevigata } \\
\text { B. cylindrica } \\
\text { B. caudata } \\
\text { B. pannosa } \\
\text { P. aff. martinii }\end{array}$ & $\begin{array}{l}\text { Lande (1948) } \\
\text { Hoffmann et al. (1978) } \\
\text { Krmpotic et al. (1972) } \\
\text { Hoffmann et al. (1978) } \\
\text { Cai et al. (2006) } \\
\text { Thuy et al. (2019) }\end{array}$ \\
\hline 82 & $(R)$-boehmeriasin $\mathrm{A}$ & B. siamensis & Luo et al. (2003) \\
\hline 83 & Cryptopleuridine & C. pleurosperma & Johns et al. (1970) \\
\hline 84 & (14a $R, 15 R$ )-hydroxycryptopleurine & B. pannosa & Cai et al. (2006) \\
\hline 85 & Boehmeriasin B & B. siamensis & Luo et al. (2003) \\
\hline 86 & Pileamartine $\mathrm{C}$ & $P$. aff. martinii & Thuy et al. (2019) \\
\hline
\end{tabular}


Table 3 continued

\begin{tabular}{llll}
\hline Compounds & Name & Origins & References \\
\hline 87 & Pileamartine D & P. aff. martinii & Thuy et al. (2019) \\
88 & Kayawongine & C. rheifolia & Saifah et al. (1983) \\
89 & Julandine & P. aff. martinii & Thuy et al. (2019) \\
\hline
\end{tabular}

* Natural PIAs presented in this Table were all isolated after the year 2000, those PIA analogues obtained before had been summarized in a few reviews (Govindachari and Viswanathan 1978; Gellert 1982; Li et al. 2001)

**All nine isolated PQA analogues up to date were presented in this Table<smiles>c1ccc2c(c1)c1c(c3ccccc32)CN2CCCC2C1</smiles>

I-1<smiles>c1ccc(C2=C(c3ccccc3)CN3CCCC3C2)cc1</smiles>

I-4<smiles>[O-][N+]12CCCC1Cc1c(c3ccccc3c3ccccc13)C2</smiles>

I-2<smiles>c1ccc2c(c1)c1c(c3ccccc32)CN2CCCCC2C1</smiles>

II-1<smiles>C1=c2c3ccccc3c(c3ccccc23)=C[N+]2=C1CCC2</smiles>

I-3<smiles>c1ccc(C2=C(c3ccccc3)CN3CCCCC3C2)cc1</smiles>

II-2

Fig. 2 Skeleton variations of the isolated PIAs (I-1-I-4) and PQAs (II-1 and II-2)

with trans or cis configuration to H-13a. Among them, compounds 16-18 $(13 \mathrm{a} R, 14 R)$ and 28-36 $(13 \mathrm{a} S, 14 R)$ possessed a trans configuration, however, compounds 19 and $20(13 \mathrm{a} R, 14 S), \mathbf{3 7}-\mathbf{3 9}(13 \mathrm{a} S, 14 S)$ were all with a cis configuration. Compound $\mathbf{3 6}$ was the first glucoside of PIAs isolated from nature, hispiloscine (40) firstly obtained with an acetoxy substitution at C-14 naturally, and tengechlorenine (40) was the first naturally occurring halogenated PIA derivative.

Nitrogen atom in PIAs could be further oxidized to yield $N$-oxides (41-66, Fig. 5). Four kinds of relative configurations between $\mathrm{H}-13 \mathrm{a}$ and $\mathrm{N}$-oxide group could be generated in PIA $N$-oxides $(10 S, 13 \mathrm{a} R$; $10 R, 13 \mathrm{a} S ; 10 R, 13 \mathrm{a} R$ and $10 S, 13 \mathrm{a} S)$. Compounds 4152 were $N$-oxides all with $10 S, 13 \mathrm{a} R$ absolute configurations and 53-60 with $10 R, 13 \mathrm{a} S$ stereochemistry. $\mathrm{H}-13 \mathrm{a}$ and $N$-oxide group in 61-66 were all cis configuration, while 61-64 were $10 R, 13 \mathrm{a} R$ and $\mathbf{6 5}, 66$ were $10 S, 13 \mathrm{a} S$. Furthermore, $N$-oxides also could be hydroxylated at $\mathrm{C}-14$ and more of them possessed the trans configuration with H-13a (47-51, 57-60), only compounds 52, 55 and $\mathbf{5 6}$ were found to be the cis configuration.

Indolizidine moiety of PIAs could undergo further dehydrogenation in ring $\mathrm{D}(\mathbf{6 7 - 6 9})$ or ring $\mathrm{E}(\mathbf{7 0})$ to give rise to dehydro- analogues (Fig. 6). These structural variations either extended the conjugated system, or increased the rigidity and planarity of the whole structure skeleton. In addition, the phenanthrene moiety could be cracked between C-4a and $4 b$ to yield seco-PIA derivatives (71-80) (Fig. 6). On the foundation of which, further oxidation also could generate on the nitrogen atom to get a $N$-oxide (79), or undergo further dehydrogenation in ring $\mathrm{D}$ to give rise to a dehydro- analogue $(\mathbf{8 0})$. 
<smiles>[R]c1cc2c3c(c4cc([R])c([R])c([R])c4c2c([R])c1[R])[C@@H]([R7])[C@@H]1CCCN1C3</smiles>

1: $\mathrm{R}_{1}=\mathrm{OCH}_{3}, \mathrm{R}_{2}=\mathrm{OCH}_{3}, \mathrm{R}_{3}=\mathrm{H}, \mathrm{R}_{4}=\mathrm{H}, \mathrm{R}_{5}=\mathrm{OCH}_{3}, \mathrm{R}_{6}=\mathrm{OCH}_{3}, \mathrm{R}_{7}=\mathrm{H}$

2: $\mathrm{R}_{1}=\mathrm{OCH}_{3}, \mathrm{R}_{2}=\mathrm{OCH}_{3}, \mathrm{R}_{3}=\mathrm{H}, \mathrm{R}_{4}=\mathrm{H}, \mathrm{R}_{5}=\mathrm{OCH}_{3}, \mathrm{R}_{6}=\mathrm{H}, \mathrm{R}_{7}=\mathrm{H}$

5: $\mathrm{R}_{1}=\mathrm{H}, \mathrm{R}_{2}=\mathrm{OCH}_{3}, \mathrm{R}_{3}=\mathrm{OCH}_{3}, \mathrm{R}_{4}=\mathrm{H}, \mathrm{R}_{5}=\mathrm{OCH}_{3}, \mathrm{R}_{6}=\mathrm{H}, \mathrm{R}_{7}=\mathrm{H}$

6: $\mathrm{R}_{1}=\mathrm{OH}, \mathrm{R}_{2}=\mathrm{OCH}_{3}, \mathrm{R}_{3}=\mathrm{OH}, \mathrm{R}_{4}=\mathrm{H}, \mathrm{R}_{5}=\mathrm{OCH}_{3}, \mathrm{R}_{6}=\mathrm{H}, \mathrm{R}_{7}=\mathrm{H}$

7: $\mathrm{R}_{1}=\mathrm{OCH}_{3}, \mathrm{R}_{2}=\mathrm{OCH}_{3}, \mathrm{R}_{3}=\mathrm{OH}, \mathrm{R}_{4}=\mathrm{OCH}_{3}, \mathrm{R}_{5}=\mathrm{OCH}_{3}, \mathrm{R}_{6}=\mathrm{H}, \mathrm{R}_{7}=\mathrm{H}$

8: $\mathrm{R}_{1}=\mathrm{H}, \mathrm{R}_{2}=\mathrm{OCH}_{3}, \mathrm{R}_{3}=\mathrm{OCH}_{3}, \mathrm{R}_{4}=\mathrm{H}, \mathrm{R}_{5}=\mathrm{OH}, \mathrm{R}_{6}=\mathrm{OCH}_{3}, \mathrm{R}_{7}=\mathrm{H}$

9: $\mathrm{R}_{1}=\mathrm{OCH}_{3}, \mathrm{R}_{2}=\mathrm{OH}, \mathrm{R}_{3}=\mathrm{H}, \mathrm{R}_{4}=\mathrm{OCH}_{3}, \mathrm{R}_{5}=\mathrm{OCH}_{3}, \mathrm{R}_{6}=\mathrm{H}, \mathrm{R}_{7}=\mathrm{H}$

10: $\mathrm{R}_{1}=\mathrm{OCH}_{3}, \mathrm{R}_{2}=\mathrm{OCH}_{3}, \mathrm{R}_{3}=\mathrm{H}, \mathrm{R}_{4}=\mathrm{OCH}_{3}, \mathrm{R}_{5}=\mathrm{OH}, \mathrm{R}_{6}=\mathrm{H}, \mathrm{R}_{7}=\mathrm{H}$

11: $\mathrm{R}_{1}=\mathrm{OCH}_{3}, \mathrm{R}_{2}=\mathrm{OCH}_{3}, \mathrm{R}_{3}=\mathrm{H}, \mathrm{R}_{4}=\mathrm{OCH}_{3}, \mathrm{R}_{5}=\mathrm{OCH}_{3}, \mathrm{R}_{6}=\mathrm{H}, \mathrm{R}_{7}=\mathrm{H}$

12: $\mathrm{R}_{1}=\mathrm{H}, \mathrm{R}_{2}=\mathrm{OCH}_{3}, \mathrm{R}_{3}=\mathrm{OCH}_{3}, \mathrm{R}_{4}=\mathrm{H}, \mathrm{R}_{5}=\mathrm{OCH}_{3}, \mathrm{R}_{6}=\mathrm{OCH}_{3}, \mathrm{R}_{7}=\mathrm{H}$

13: $R_{1}=H, R_{2}=O_{3}, R_{3}=H, R_{4}=H, R_{5}=O H, R_{6}=O_{3}, R_{7}=H$

14: $\mathrm{R}_{1}=\mathrm{OCH}_{3}, \mathrm{R}_{2}=\mathrm{OCH}_{3}, \mathrm{R}_{3}=\mathrm{H}, \mathrm{R}_{4}=\mathrm{H}, \mathrm{R}_{5}=\mathrm{OH}, \mathrm{R}_{6}=\mathrm{H}, \mathrm{R}_{7}=\mathrm{H}$

15: $\mathrm{R}_{1}=\mathrm{OCH}_{3}, \mathrm{R}_{2}=\mathrm{OCH}_{3}, \mathrm{R}_{3}=\mathrm{H}, \mathrm{R}_{4}=\mathrm{H}, \mathrm{R}_{5}=\mathrm{OCH}_{3}, \mathrm{R}_{6}=\mathrm{OH}, \mathrm{R}_{7}=\mathrm{H}$

16: $\mathrm{R}_{1}=\mathrm{OH}, \mathrm{R}_{2}=\mathrm{OCH}_{3}, \mathrm{R}_{3}=\mathrm{H}, \mathrm{R}_{4}=\mathrm{H}, \mathrm{R}_{5}=\mathrm{OCH}_{3}, \mathrm{R}_{6}=\mathrm{OCH}_{3}, \mathrm{R}_{7}=\mathrm{OH}$

17: $\mathrm{R}_{1}=\mathrm{OCH}_{3}, \mathrm{R}_{2}=\mathrm{OCH}_{3}, \mathrm{R}_{3}=\mathrm{H}, \mathrm{R}_{4}=\mathrm{H}, \mathrm{R}_{5}=\mathrm{OCH}_{3}, \mathrm{R}_{6}=\mathrm{OCH}_{3}, \mathrm{R}_{7}=\mathrm{OH}$

18: $\mathrm{R}_{1}=\mathrm{H}, \mathrm{R}_{2}=\mathrm{OCH}_{3}, \mathrm{R}_{3}=\mathrm{H}, \mathrm{R}_{4}=\mathrm{H}, \mathrm{R}_{5}=\mathrm{OCH}_{3}, \mathrm{R}_{6}=\mathrm{OCH}_{3}, \mathrm{R}_{7}=\mathrm{OH}$<smiles>[R]c1c(OC)ccc2c3c(c4cc5c(cc4c12)OCO5)C[C@@H]1CCCN1C3</smiles>

3: $\mathrm{R}=\mathrm{OCH}_{3}$

4: $\mathrm{R}=\mathrm{H}$

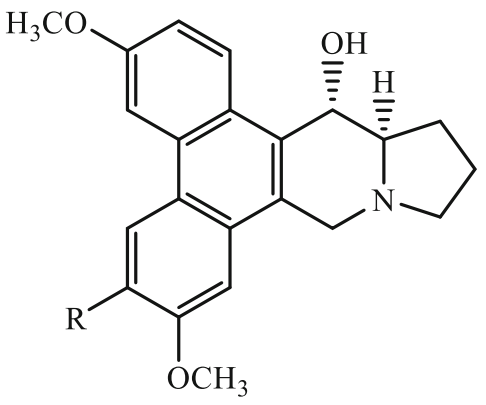

19: $\mathrm{R}=\mathrm{OCH}_{3}$

20: $\mathrm{R}=\mathrm{OH}$

Fig. 3 Structures of isolated PIAs with $13 \mathrm{a} R$ - configuration

Comparing with PIAs, fewer naturally occurring PQAs were isolated. Up to now, only nine PQAs analogues (81-89) were obtained from the Lauraceae, Vitaceae and Urticaceae family of plants (Fig. 7). Their skeleton variations mainly were revealed by the stereochemistry of C-14a (81 and $\mathbf{8 8})$ and the secophenanthrene moiety $(\mathbf{8 9})$.

\section{Key spectroscopic characteristics of PIAs and PQAs}

UV spectra could be used to differentiate those dehydro-analogues with an aromatic D-ring (67-69, Fig. 6). Their conjugated chromophoric system between the phenanthrene moiety and D-ring in the skeleton caused a main maximum absorption $\left(\lambda_{\max }\right)$ at approximately $280 \mathrm{~nm}$ with a shoulder peak at 255-260 $\mathrm{nm}$ in their UV spectra, while $\lambda_{\max }$ of other
PIAs could be recorded at $260 \mathrm{~nm}$ with a shoulder at 280-285 nm, which indicated the D-ring was saturated (Cui et al. 2004).

Except those dehydro- analogues, such as compounds 67-70, almost all the PIA and PQA derivatives could be involved in the stereochemistry determination of C-13a (for PIAs) or C-14a (for PQAs). With regards to the configuration at $\mathrm{C}-13 \mathrm{a}$, it appeared that PIAs with the $S$ - configuration exhibited a positive optical rotation, the opposite was observed with the $R$ configuration. Variation in the substituents and substitution pattern in both the phenanthrene and indolizidine moieties did not seem to significantly alter the optical rotational properties of the alkaloids (Yap et al. 2015). Furthermore, CD or ORD technique also was used to resolve this problem by the following rule: the positive optical rotation with the negative Cotton effect at approximately $\lambda 260 \mathrm{~nm}$ corresponding to the $\mathrm{C}-13 \mathrm{a} S$ configuration, however, the negative 
<smiles>[R]c1cc2c3c(c4cc([R])c([R])c([R])c4c2c([R])c1[R])C([R7])[C@H]1CCCN1C3</smiles>

21: $\mathrm{R}_{1}=\mathrm{OCH}_{3}, \mathrm{R}_{2}=\mathrm{OCH}_{3}, \mathrm{R}_{3}=\mathrm{H}, \mathrm{R}_{4}=\mathrm{H}, \mathrm{R}_{5}=\mathrm{OCH}_{3}, \mathrm{R}_{6}=\mathrm{OCH}_{3}, \mathrm{R}_{7}=\mathrm{H}$

22: $\mathrm{R}_{1}=\mathrm{OH}, \mathrm{R}_{2}=\mathrm{OCH}_{3}, \mathrm{R}_{3}=\mathrm{H}, \mathrm{R}_{4}=\mathrm{H}, \mathrm{R}_{5}=\mathrm{OH}, \mathrm{R}_{6}=\mathrm{OCH}_{3}, \mathrm{R}_{7}=\mathrm{H}$

23: $\mathrm{R}_{1}=\mathrm{OCH}_{3}, \mathrm{R}_{2}=\mathrm{OCH}_{3}, \mathrm{R}_{3}=\mathrm{H}, \mathrm{R}_{4}=\mathrm{OCH}_{3}, \mathrm{R}_{5}=\mathrm{OH}, \mathrm{R}_{6}=\mathrm{H}, \mathrm{R}_{7}=\mathrm{H}$

24: $\mathrm{R}_{1}=\mathrm{OCH}_{3}, \mathrm{R}_{2}=\mathrm{OCH}_{3}, \mathrm{R}_{3}=\mathrm{H}, \mathrm{R}_{4}=\mathrm{OCH}_{3}, \mathrm{R}_{5}=\mathrm{OCH}_{3}, \mathrm{R}_{6}=\mathrm{H}, \mathrm{R}_{7}=\mathrm{H}$

25: $\mathrm{R}_{1}=\mathrm{H}, \mathrm{R}_{2}=\mathrm{OCH}_{3}, \mathrm{R}_{3}=\mathrm{H}, \mathrm{R}_{4}=\mathrm{H}, \mathrm{R}_{5}=\mathrm{OCH}_{3}, \mathrm{R}_{6}=\mathrm{OCH}_{3}, \mathrm{R}_{7}=\mathrm{H}$

26: $\mathrm{R}_{1}=\mathrm{H}, \mathrm{R}_{2}=\mathrm{OH}, \mathrm{R}_{3}=\mathrm{OCH}_{3}, \mathrm{R}_{4}=\mathrm{H}, \mathrm{R}_{5}=\mathrm{OCH}_{3}, \mathrm{R}_{6}=\mathrm{OCH}_{3}, \mathrm{R}_{7}=\mathrm{H}$

27: $\mathrm{R}_{1}=\mathrm{H}, \mathrm{R}_{2}=\mathrm{OCH}_{3}, \mathrm{R}_{3}=\mathrm{OCH}_{3}, \mathrm{R}_{4}=\mathrm{H}, \mathrm{R}_{5}=\mathrm{OCH}_{3}, \mathrm{R}_{6}=\mathrm{OCH}_{3}, \mathrm{R}_{7}=\mathrm{H}$

28: $\mathrm{R}_{1}=\mathrm{OH}, \mathrm{R}_{2}=\mathrm{OCH}_{3}, \mathrm{R}_{3}=\mathrm{H}, \mathrm{R}_{4}=\mathrm{H}, \mathrm{R}_{5}=\mathrm{OH}, \mathrm{R}_{6}=\mathrm{OCH}_{3}, \mathrm{R}_{7}=\mathrm{OH}$

29: $\mathrm{R}_{1}=\mathrm{H}, \mathrm{R}_{2}=\mathrm{OH}, \mathrm{R}_{3}=\mathrm{H}, \mathrm{R}_{4}=\mathrm{H}, \mathrm{R}_{5}=\mathrm{OH}, \mathrm{R}_{6}=\mathrm{OCH}_{3}, \mathrm{R}_{7}=\mathrm{OH}$

30: $\mathrm{R}_{1}=\mathrm{H}, \mathrm{R}_{2}=\mathrm{OCH}_{3}, \mathrm{R}_{3}=\mathrm{H}, \mathrm{R}_{4}=\mathrm{H}, \mathrm{R}_{5}=\mathrm{OH}, \mathrm{R}_{6}=\mathrm{OCH}_{3}, \mathrm{R}_{7}=\mathrm{OH}$

31: $\mathrm{R}_{1}=\mathrm{H}, \mathrm{R}_{2}=\mathrm{OH}, \mathrm{R}_{3}=\mathrm{H}, \mathrm{R}_{4}=\mathrm{H}, \mathrm{R}_{5}=\mathrm{OCH}_{3}, \mathrm{R}_{6}=\mathrm{OCH}_{3}, \mathrm{R}_{7}=\mathrm{OH}$

32: $\mathrm{R}_{1}=\mathrm{H}, \mathrm{R}_{2}=\mathrm{OCH}_{3}, \mathrm{R}_{3}=\mathrm{H}, \mathrm{R}_{4}=\mathrm{H}, \mathrm{R}_{5}=\mathrm{OCH}_{3}, \mathrm{R}_{6}=\mathrm{OCH}_{3}, \mathrm{R}_{7}=\mathrm{OH}$

33: $\mathrm{R}_{1}=\mathrm{H}, \mathrm{R}_{2}=\mathrm{OH}, \mathrm{R}_{3}=\mathrm{OCH}_{3}, \mathrm{R}_{4}=\mathrm{H}, \mathrm{R}_{5}=\mathrm{OCH}_{3}, \mathrm{R}_{6}=\mathrm{OCH}_{3}, \mathrm{R}_{7}=\mathrm{OH}$<smiles>[R]c1cc2c(cc1OC)c1c(c3ccc(OC)cc32)[C@H](O)[C@@H]2CCC(=O)N2C1</smiles>

34: $\mathrm{R}=\mathrm{OH}$

35: $\mathrm{R}=\mathrm{OCH}_{3}$<smiles>[R]c1ccc2c3c(c4cc(OC)c(OC)cc4c2c1)CN1CCC[C@@H]1[C@H]3[R2]</smiles>

37: $\mathrm{R}_{1}=\mathrm{OCH}_{3}, \mathrm{R}_{2}=\mathrm{OH}$

38: $\mathrm{R}_{1}=\mathrm{OH}, \mathrm{R}_{2}=\mathrm{OH}$

39: $\mathrm{R}_{1}=\mathrm{OCH}_{3}, \mathrm{R}_{2}=\mathrm{OCOCH}_{3}$

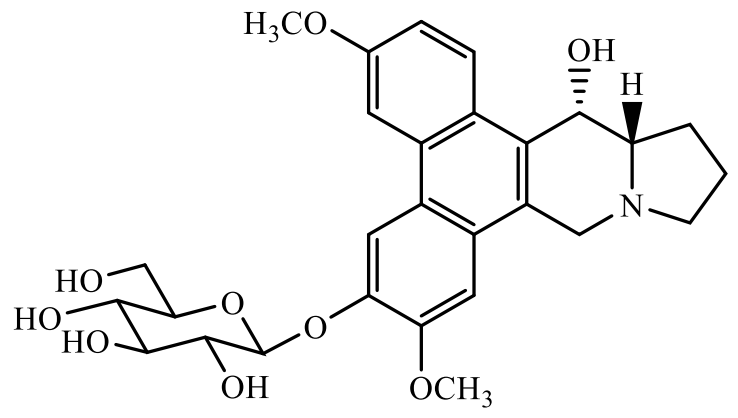

36<smiles>COc1cc2c3c(c4ccc(OC)c(Cl)c4c2cc1OC)CN1CCC[C@H]1C3</smiles>

40

Fig. 4 Structures of isolated PIAs with $13 \mathrm{a} S$ - configuration

optical rotation and a positive Cotton effect for alkaloids with the $13 \mathrm{a} R$ configuration (Damu et al. 2009). These conclusions also could be applied to PQAs for the stereochemistry determination of C-14a (Cai et al. 2006; Thuy et al. 2019). Based on the stereochemistry of C-13a, the relative configuration of the hydroxy group at $\mathrm{C}-14$ could be determined by the coupling constant $(J)$ or NOE between $\mathrm{H}-14$ and
H-13a (Lee et al. 2011; Yap et al. 2015; Chen et al. 2019a). For $N$-oxides, the resonance due to $\mathrm{H}-13 \mathrm{a}$ at approximately $\delta 3.5$ was indicative of the trans-fused indolizidine ring junction, however, $c$ is-fused junction resulted in the resonance of $\mathrm{H}-13 \mathrm{a}$ at approximately $\delta$ 4.0 (Damu et al. 2005, 2009; Chen et al. 2016).

In addition, mass spectrometry (MS) could reveal the relationship between the interesting fragmentation 
<smiles>[R]C[C@]12CCC[N+]1([O-])Cc1c(c3cc([R])c([R])c([R])c3c3c([R])c([R])c([R])cc13)[C@H]2[R7]</smiles>

41: $\mathrm{R}_{1}=\mathrm{OCH}_{3}, \mathrm{R}_{2}=\mathrm{OCH}_{3}, \mathrm{R}_{3}=\mathrm{H}, \mathrm{R}_{4}=\mathrm{H}, \mathrm{R}_{5}=\mathrm{OCH}_{3}, \mathrm{R}_{6}=\mathrm{H}, \mathrm{R}_{7}=\mathrm{H}$ 42: $\mathrm{R}_{1}=\mathrm{OCH}_{3}, \mathrm{R}_{2}=\mathrm{OCH}_{3}, \mathrm{R}_{3}=\mathrm{H}, \mathrm{R}_{4}=\mathrm{OCH}_{3}, \mathrm{R}_{5}=\mathrm{OCH}_{3}, \mathrm{R}_{6}=\mathrm{H}, \mathrm{R}_{7}=\mathrm{H}$ 43: $\mathrm{R}_{1}=\mathrm{H}, \mathrm{R}_{2}=\mathrm{OCH}_{3}, \mathrm{R}_{3}=\mathrm{OCH}_{3}, \mathrm{R}_{4}=\mathrm{H}, \mathrm{R}_{5}=\mathrm{OCH}_{3}, \mathrm{R}_{6}=\mathrm{OCH}_{3}, \mathrm{R}_{7}=\mathrm{H}$ 44: $\mathrm{R}_{1}=\mathrm{OCH}_{3}, \mathrm{R}_{2}=\mathrm{OCH}_{3}, \mathrm{R}_{3}=\mathrm{H}, \mathrm{R}_{4}=\mathrm{OCH}_{3}, \mathrm{R}_{5}=\mathrm{OH}, \mathrm{R}_{6}=\mathrm{H}, \mathrm{R}_{7}=\mathrm{H}$ 45: $\mathrm{R}_{1}=\mathrm{H}, \mathrm{R}_{2}=\mathrm{OCH}_{3}, \mathrm{R}_{3}=\mathrm{OCH}_{3}, \mathrm{R}_{4}=\mathrm{H}, \mathrm{R}_{5}=\mathrm{OCH}_{3}, \mathrm{R}_{6}=\mathrm{H}, \mathrm{R}_{7}=\mathrm{H}$ 46: $\mathrm{R}_{1}=\mathrm{OCH}_{3}, \mathrm{R}_{2}=\mathrm{OCH}_{3}, \mathrm{R}_{3}=\mathrm{H}, \mathrm{R}_{4}=\mathrm{H}, \mathrm{R}_{5}=\mathrm{OCH}_{3}, \mathrm{R}_{6}=\mathrm{OCH}_{3}, \mathrm{R}_{7}=\mathrm{H}$ 47: $\mathrm{R}_{1}=\mathrm{OCH}_{3}, \mathrm{R}_{2}=\mathrm{OCH}_{3}, \mathrm{R}_{3}=\mathrm{H}, \mathrm{R}_{4}=\mathrm{H}, \mathrm{R}_{5}=\mathrm{OCH}_{3}, \mathrm{R}_{6}=\mathrm{H}, \mathrm{R}_{7}=\mathrm{OH}$ 48: $\mathrm{R}_{1}=\mathrm{OH}, \mathrm{R}_{2}=\mathrm{OCH}_{3}, \mathrm{R}_{3}=\mathrm{H}, \mathrm{R}_{4}=\mathrm{H}, \mathrm{R}_{5}=\mathrm{OH}, \mathrm{R}_{6}=\mathrm{H}, \mathrm{R}_{7}=\mathrm{OH}$ 49: $\mathrm{R}_{1}=\mathrm{H}, \mathrm{R}_{2}=\mathrm{OCH}_{3}, \mathrm{R}_{3}=\mathrm{H}, \mathrm{R}_{4}=\mathrm{H}, \mathrm{R}_{5}=\mathrm{OCH}_{3}, \mathrm{R}_{6}=\mathrm{OCH}_{3}, \mathrm{R}_{7}=\mathrm{OH}$ 50: $\mathrm{R}_{1}=\mathrm{OCH}_{3}, \mathrm{R}_{2}=\mathrm{OCH}_{3}, \mathrm{R}_{3}=\mathrm{H}, \mathrm{R}_{4}=\mathrm{H}, \mathrm{R}_{5}=\mathrm{OCH}_{3}, \mathrm{R}_{6}=\mathrm{OCH}_{3}, \mathrm{R}_{7}=\mathrm{OH}$<smiles></smiles><smiles>COc1cc2c3c(c4ccc5c(c4c2cc1OC)OCO5)[C@H](O)[C@H]1CCC[N@+]1([O])C3</smiles><smiles>COc1cc2c3c(c4ccc(O)c(OC)c4c2cc1OC)[C@@H](O)[C@H]1CCC[N@+]1([O-])C3</smiles>

52<smiles></smiles>

53: $\mathrm{R}_{1}=\mathrm{OCH}_{3}, \mathrm{R}_{2}=\mathrm{OCH}_{3}, \mathrm{R}_{3}=\mathrm{H}, \mathrm{R}_{4}=\mathrm{H}, \mathrm{R}_{5}=\mathrm{OCH}_{3}, \mathrm{R}_{6}=\mathrm{OCH}_{3}, \mathrm{R}_{7}=\mathrm{H}$

54: $\mathrm{R}_{1}=\mathrm{H}, \mathrm{R}_{2}=\mathrm{OCH}_{3}, \mathrm{R}_{3}=\mathrm{H}, \mathrm{R}_{4}=\mathrm{H}, \mathrm{R}_{5}=\mathrm{OCH}_{3}, \mathrm{R}_{6}=\mathrm{OCH}_{3}, \mathrm{R}_{7}=\mathrm{H}$

55: $\mathrm{R}_{1}=\mathrm{H}, \mathrm{R}_{2}=\mathrm{OH}, \mathrm{R}_{3}=\mathrm{H}, \mathrm{R}_{4}=\mathrm{H}, \mathrm{R}_{5}=\mathrm{OH}, \mathrm{R}_{6}=\mathrm{OCH}_{3}, \mathrm{R}_{7}=\beta-\mathrm{OH}$

56: $\mathrm{R}_{1}=\mathrm{OH}, \mathrm{R}_{2}=\mathrm{OCH}_{3}, \mathrm{R}_{3}=\mathrm{H}, \mathrm{R}_{4}=\mathrm{H}, \mathrm{R}_{5}=\mathrm{OCH}_{3}, \mathrm{R}_{6}=\mathrm{OCH}_{3}, \mathrm{R}_{7}=\beta-\mathrm{OH}$

57: $\mathrm{R}_{1}=\mathrm{OCH}_{3}, \mathrm{R}_{2}=\mathrm{OCH}_{3}, \mathrm{R}_{3}=\mathrm{H}, \mathrm{R}_{4}=\mathrm{H}, \mathrm{R}_{5}=\mathrm{OCH}_{3}, \mathrm{R}_{6}=\mathrm{OCH}_{3}, \mathrm{R}_{7}=\alpha-\mathrm{OH}$

59: $\mathrm{R}_{1}=\mathrm{OCH}_{3}, \mathrm{R}_{2}=\mathrm{OCH}_{3}, \mathrm{R}_{3}=\mathrm{OCH}_{3}, \mathrm{R}_{4}=\mathrm{H}, \mathrm{R}_{5}=\mathrm{OCH}_{3}, \mathrm{R}_{6}=\mathrm{OCH}_{3}, \mathrm{R}_{7}=\alpha-\mathrm{OH}$

60: $\mathrm{R}_{1}=\mathrm{H}, \mathrm{R}_{2}=\mathrm{OCH}_{3}, \mathrm{R}_{3}=\mathrm{OCH}_{3}, \mathrm{R}_{4}=\mathrm{H}, \mathrm{R}_{5}=\mathrm{OCH}_{3}, \mathrm{R}_{6}=\mathrm{OCH}_{3}, \mathrm{R}_{7}=\alpha-\mathrm{OH}$<smiles></smiles>

61: $\mathrm{R}_{1}=\mathrm{H}, \mathrm{R}_{2}=\mathrm{OCH}_{3}, \mathrm{R}_{3}=\mathrm{OCH}_{3}, \mathrm{R}_{4}=\mathrm{H}, \mathrm{R}_{5}=\mathrm{OCH}_{3}, \mathrm{R}_{6}=\mathrm{OCH}_{3}, \mathrm{R}_{7}=\mathrm{H}$

62: $\mathrm{R}_{1}=\mathrm{H}, \mathrm{R}_{2}=\mathrm{OCH}_{3}, \mathrm{R}_{3}=\mathrm{H}, \mathrm{R}_{4}=\mathrm{H}, \mathrm{R}_{5}=\mathrm{OH}, \mathrm{R}_{6}=\mathrm{OCH}_{3}, \mathrm{R}_{7}=\alpha-\mathrm{OH}$

63: $\mathrm{R}_{1}=\mathrm{H}, \mathrm{R}_{2}=\mathrm{OH}, \mathrm{R}_{3}=\mathrm{OCH}_{3}, \mathrm{R}_{4}=\mathrm{H}, \mathrm{R}_{5}=\mathrm{OCH}_{3}, \mathrm{R}_{6}=\mathrm{OCH}_{3}, \mathrm{R}_{7}=\alpha-\mathrm{OH}$

64: $\mathrm{R}_{1}=\mathrm{H}, \mathrm{R}_{2}=\mathrm{OCH}_{3}, \mathrm{R}_{3}=\mathrm{H}, \mathrm{R}_{4}=\mathrm{H}, \mathrm{R}_{5}=\mathrm{OCH}_{3}, \mathrm{R}_{6}=\mathrm{OCH}_{3}, \mathrm{R}_{7}=\beta-\mathrm{OH}$<smiles>[R2]c1cc2c3c(c4cc(OC)c(OC)cc4c2c([R])c1OC)C[N@+]1([O-])CCC[C@H]1C3</smiles>

65: $\mathrm{R}_{1}=\mathrm{H}, \mathrm{R}_{2}=\mathrm{OCH}_{3}$

66: $\mathrm{R}_{1}=\mathrm{OCH}_{3}, \mathrm{R}_{2}=\mathrm{H}$ 
\Fig. 5 Structures of isolated PIAs $N$-oxides

behavior and the molecular structure. Commonly, PIAs with a saturated D-ring always mainly produced two fragment ions via the retro-Diels-Alder (RDA) cleavage reactions. The fragmentation behavior in the<smiles>[R]c1cc2c(cc1[R])c1cc3[n+](cc1c1cc(OC)c([R])cc21)CCC3</smiles>

67: $\mathrm{R}_{1}=\mathrm{H}, \mathrm{R}_{2}=\mathrm{OCH}_{3}, \mathrm{R}_{3}=\mathrm{OCH}_{3}$

68: $\mathrm{R}_{1}=\mathrm{OCH}_{3}, \mathrm{R}_{2}=\mathrm{OCH}_{3}, \mathrm{R}_{3}=\mathrm{OCH}_{3}$

69: $\mathrm{R}_{1}=\mathrm{OCH}_{3}, \mathrm{R}_{2}=\mathrm{OCH}_{3}, \mathrm{R}_{3}=\mathrm{H}$<smiles>[R]c1ccc(C2=C(c3cc([R])c([R])c([R])c3)C[C@@H]3CCCN3C2)cc1[R]</smiles>

71: $\mathrm{R}_{1}=\mathrm{OCH}_{3}, \mathrm{R}_{2}=\mathrm{OCH}_{3}, \mathrm{R}_{3}=\mathrm{H}, \mathrm{R}_{4}=\mathrm{OCH}_{3}, \mathrm{R}_{5}=\mathrm{H}$

72: $\mathrm{R}_{1}=\mathrm{OCH}_{3}, \mathrm{R}_{2}=\mathrm{OCH}_{3}, \mathrm{R}_{3}=\mathrm{H}, \mathrm{R}_{4}=\mathrm{OH}, \mathrm{R}_{5}=\mathrm{H}$

73: $\mathrm{R}_{1}=\mathrm{OCH}_{3}, \mathrm{R}_{2}=\mathrm{OCH}_{3}, \mathrm{R}_{3}=\mathrm{OH}, \mathrm{R}_{4}=\mathrm{OCH}_{3}, \mathrm{R}_{5}=\mathrm{OCH}_{3}$<smiles>COc1ccc(C2=C(c3ccc(O)cc3)C[N@@+]3([O-])CCC[C@H]3C2)cc1OC</smiles>

79 positive $\mathrm{FAB}^{-M S^{\mathrm{n}}}$ or $\mathrm{ESI}^{-M S^{\mathrm{n}}}$ depended on the protonated position at the nitrogen atom or in the aromatic ring. When the nitrogen atom was protonated, the RDA cleavage easily produced the peak of the protonated dihydropyrrole $\left(_{\mathrm{H}^{+}{ }^{+}>}>, m / z, 70\right)$, while when the $\mathrm{H}^{+}$was added to the aromatic ring, the ion corresponding to the substituted phenanthrene moiety<smiles>[R]c1ccc(C2=C(c3ccc([R])c([R4])c3)CN3CCC[C@H]3C2)cc1[R]</smiles>

74: $\mathrm{R}_{1}=\mathrm{H}, \mathrm{R}_{2}=\mathrm{OCH}_{3}, \mathrm{R}_{3}=\mathrm{OH}, \mathrm{R}_{4}=\mathrm{OCH}_{3}$

75: $\mathrm{R}_{1}=\mathrm{H}, \mathrm{R}_{2}=\mathrm{OCH}_{3}, \mathrm{R}_{3}=\mathrm{OCH}_{3}, \mathrm{R}_{4}=\mathrm{OCH}_{3}$

76: $\mathrm{R}_{1}=\mathrm{OCH}_{3}, \mathrm{R}_{2}=\mathrm{OCH}_{3}, \mathrm{R}_{3}=\mathrm{OH}, \mathrm{R}_{4}=\mathrm{OCH}_{3}$

77: $\mathrm{R}_{1}=\mathrm{OCH}_{3}, \mathrm{R}_{2}=\mathrm{OCH}_{3}, \mathrm{R}_{3}=\mathrm{OCH}_{3}, \mathrm{R}_{4}=\mathrm{OCH}_{3}$ 78: $\mathrm{R}_{1}=\mathrm{OCH}_{3}, \mathrm{R}_{2}=\mathrm{OH}, \mathrm{R}_{3}=\mathrm{OH}, \mathrm{R}_{4}=\mathrm{OCH}_{3}$<smiles>COc1ccc(-c2c[n+]3c(cc2-c2ccc(OC)c(OC)c2)CCC3)cc1</smiles>

80

Fig. 6 Structures of isolated dehydro- (67-70) and seco- (71-80) PIAs 
Fig. 7 Structures of isolated PQAs<smiles>COc1ccc2c3c(c4cc(OC)c(OC)cc4c2c1)C[C@@H]1CCCCN1C3</smiles><smiles>COc1ccc2c3c(c4cc5c(cc4c2c1)OCO5)CN1C[C@H](O)CC[C@H]1C3</smiles><smiles>COc1ccc2c3c(c4cc(OC)c(OC)cc4c2c1)[C@H](O)C1CCCCN1C3</smiles>

84<smiles>COc1ccc2c3c(c4cc(OC)c(O)cc4c2c1)C[C@@H]1CCCCN1C3</smiles>

87<smiles>COc1ccc2c3c(c4cc(OC)c(O)cc4c2c1)CN1CCCC[C@H]1C3</smiles>

85<smiles>COc1ccc2c3c(c4ccc(OC)c(OC)c4c2c1)C[C@@H]1CCCCN1C3</smiles>

86<smiles>CCCCCCCOc1ccc([C@@H]2C[C@H]3CCCCN3C[C@H]2c2ccc(OC)c(OC)c2)cc1</smiles>

88<smiles>COc1ccc(C2=C(c3ccc(OC)c(OC)c3)C[C@@H]3CCCCN3C2)cc1</smiles>

89
$\left([\mathrm{M}+\mathrm{H}-69]^{+}\right)$was preferentially formed. Accordingly, the ion of protonated oxidized ( $N$-oxide) dihydropyrrole $\left({ }_{0}, N^{+}>, m / z\right.$ 86) or $[\mathrm{M}+\mathrm{H}-87]^{+}$ could be derived from PIA $N$-oxides via the RDA cleavage reaction, however, those quaternary PIA analogues, such as compounds 67-69, could easily produce the molecular ions $[\mathrm{M}]^{+}$, while those fragments arose from the RDA cleavage were not observed because of the aromatic D-ring. For PIAs with a hydroxyl group substituted at $\mathrm{C}-14$, the $[\mathrm{M}+\mathrm{H}]^{+}$ion was easily dehydrated to give an $\left[\mathrm{M}+\mathrm{H}-\mathrm{H}_{2} \mathrm{O}\right]^{+}$ion, while the relative abundances of $[\mathrm{M}+\mathrm{H}-69]^{+}$or $[\mathrm{M}+\mathrm{H}-87]^{+}(N$-oxide $)$ ions produced by the RDA cleavage reaction became lower since the relevant reactions were not as competitive (Xiang et al. 2002; Cui et al. 2004). Collisioninduced dissociation spectra of tylophoridicine C (62) by the positive FAB-MS might typically interpret the primary fragmentation pathway of PIAs (Fig. 8, Xiang et al. 2002). Based on the fragmentation behavior of MS, liquid chromatography combining with MS and UV techniques had been applied on line successfully to detect PIA derivatives or metabolites in the crude extract of herbal medicine (Cui et al. 2004), rat urine (Tian et al. 2012) and rat plasma (Yu et al. 2015).

\section{Biological activities of PIAs and PQAs}

New advance on anticancer activity

The favorable study on anticancer activity after the year 2015 was the isolation of PIA analogues from $T$. atrofolliculata as the hypoxia induced factor 1 (HIF-1) inhibitor (Chen et al. 2016, 2019a). HIF-1 plays a significant role in the adaption and survival of cancer cells under hypoxic conditions (Nagle and Zhou 2006). Among those 23 isolated PIAs, compounds 22, 28, 29, 30, 31, 32, 37, 48, 49, 62 (Chen et al. 2016) and 6-O- $\beta$-D-glucopyranosyl-tylophorinidine (36, Chen et al. 2019a), exhibited potent HIF-1 inhibitory activity in low nanomolar level with $\mathrm{IC}_{50}$ values ranging from 3 to $69 \mathrm{nM}$, which was more potent than digoxin, the positive control ( $\left.\mathrm{IC}_{50} 302 \mathrm{nM}\right)$. Compounds 30, 31 and 49 exhibited the most potent activity with $\mathrm{IC}_{50}$ values $<10 \mathrm{nM}$ (Chen et al. 2016). Inhibitory results indicated that substitution types and 


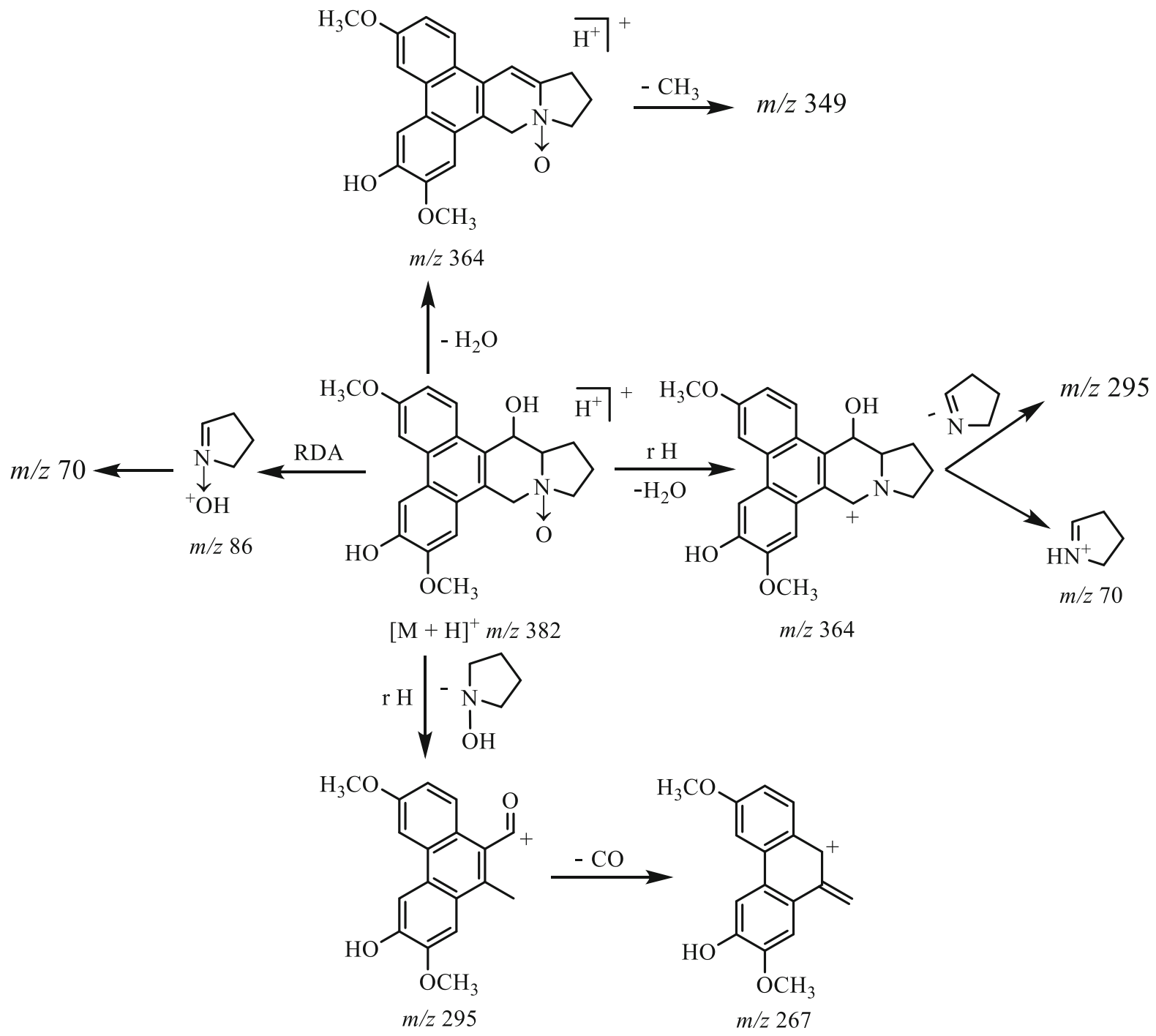

Fig. 8 Fragmentation pathway proposed to account for the CID spectra of tylophoridicine C (62) by positive ion FAB-MS. The structure of compound $\mathbf{6 2}$ was firstly elucidated to be $(13 \mathrm{a} S, 14 S)$-6,14-dihydroxy-3,7-

patterns on the phenanthrene unit (hydroxy and methoxy groups) and the indolizidine ring (amide and $N$-oxides) could influence the bioactivity. The favorable structure-activity relationship (SAR) possibly was established in Fig. 9 based on the bioassay results (Chen et al. 2016, 2019a).

In addition, $(R)$-antofine (2) was firstly reported to effectively inhibited the proliferation of Met-mutated Caki-1 cells, which were resistant to well-known Met tyrosine kinase inhibitors. It could negatively regulated Met endosomal signaling in renal cancer cells dimethoxyphenanthroindolizidine $\mathrm{N}$-oxide from $T$. atrofolliculata, also named as tylophoridicine $\mathrm{C}$ (Huang et al. 2004), while its name of 3,6,7-trimethoxy-10-oxyphenanthroindolizidine for FAB-MS in the reference was incorrect (Xiang et al. 2002)

and consequently inhibited the nuclear translocation of STAT3 both in vitro and in vivo (Song et al. 2015). Another two new seco-PIA analogues, fistulopsines A (73, $\mathrm{GI}_{50} 3.45$ and $\left.2.37 \mu \mathrm{M}\right)$ and $\mathrm{B}\left(\mathbf{7 8}, \mathrm{GI}_{50} 7.04\right.$ and $5.96 \mu \mathrm{M})$ showed in vitro growth inhibitory activity in breast carcinoma cell MCF7 and colon carcinoma cell HCT 116 cell lines, by arresting cells in G1 phase without induction of apoptosis (Yap et al. 2016). The rare chlorinated derivative tengechlorenine (40) showed pronounced in vitro cytotoxic activity against three breast cancer cell lines tested (MDA-MB-468, 


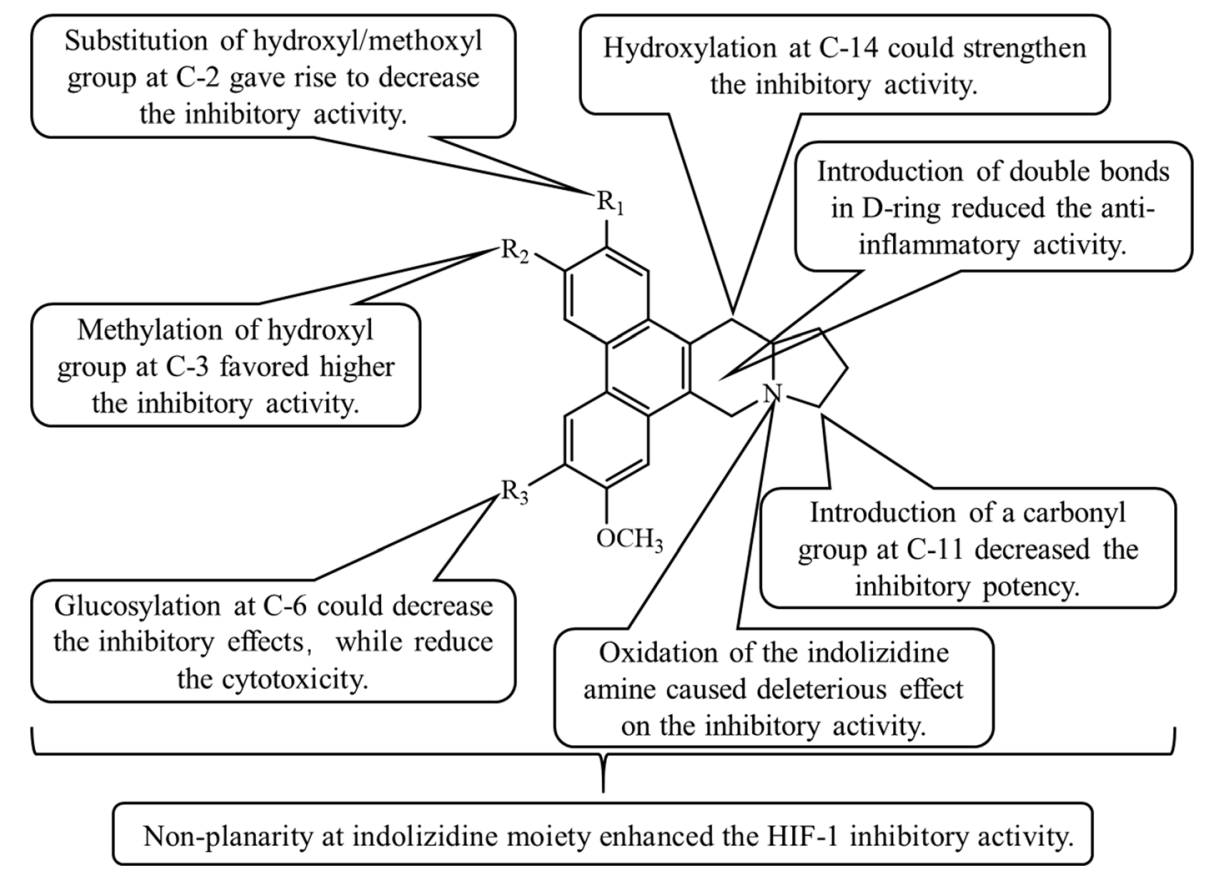

Fig. 9 Possible SARs on the HIF-1 inhibitory activity of PIAs analogues. SARs were deduced entirely from the HIF-1 inhibitory results of natural PIA analogues isolated from T. atrofolliculata (Chen et al. 2016, 2019a)

MDA-MB-231, and MCF7; $\mathrm{IC}_{50}$ values of $0.038,0.48$ and $0.91 \mu \mathrm{M})($ Al-Khdhairawi et al. 2017).

Four PQA analogues (86-89) including two new ones (86 and $\mathbf{8 7}$ ) were evaluated for their cytotoxicity against four cancer cell lines: KB (mouth epidermal carcinoma cells), HepG-2 (human liver hepatocellular carcinoma cells), LU-1 (human lung adenocarcinoma cells), and MCF-7 (human breast cancer cells). Pileamartine D (87) showed strong and selective inhibition toward $\mathrm{KB}$ and HepG-2 cells with $\mathrm{IC}_{50}$ values of 25 and $27 \mathrm{nM}$, respectively. Pileamartine $\mathrm{C}$ (86), julandine (89), and cryptopleurine (88) exhibited cytotoxicity against these four tested cancer cell lines with $\mathrm{IC}_{50}$ values less than $1 \mu \mathrm{M}$. PQA derivatives with the seco skeleton could reduce the cytotoxicity ( $88 \mathrm{vs.}$ 89), while the free hydroxyl group at C-3 seemed to be critical for the bioactivity because pileamartine D (87) was more active than $S$-cryptopleurine against 3 cancer cell lines (KB, HepG-2 and LU-1) (Thuy et al. 2019).

Anti-inflammatory activity

Anti-inflammatory activities of four naturally occurring PIAs, including $R$-tylophorine (1), $R$-antofine (2),
ficuseptine-A (59), and dehydrotylophorine (68), were all examined on the Raw264.7 macrophage cell model in vitro induced by LPS/IFN $\gamma$ by detecting the suppression of different proinflammatory cytokines such as NO, TNF $\alpha$, etc., they all exhibited potent suppression of NO in nanomolar or micromolar level (Yang et al. 2006, 2007; Min et al. 2010; Chou et al. 2017). Furthermore, $R$-antofine (2) was also indicated it could attenuate the metabolic disorders induced by inflammation via AMP-activated protein kinase as one possible molecular mechanism (Chou et al. 2017).

Based on the anti-inflammatory activities of these four naturally occurring PIAs, a series of synthesized derivatives of $R$-tylophorine (1) and $R$-antofine (2) were applied to the anti-inflammatory activity, and the possible structural requirements for the inhibition of NO production were suggested (Fig. 10). Tylophorine (1) exhibited much better activities than its framework (s-1) indicated the four methoxy groups at C-2, 3, 6 and 7 positions of the phenanthrene moiety contributed significant potency (Yang et al. 2007). However, inhibition of NO production of a series of synthesized derivatives of antofine (2) confirmed the methoxy group at C-2 and C-6 might be mandatory, and the methoxy group at $\mathrm{C}-7$ with an insignificant 

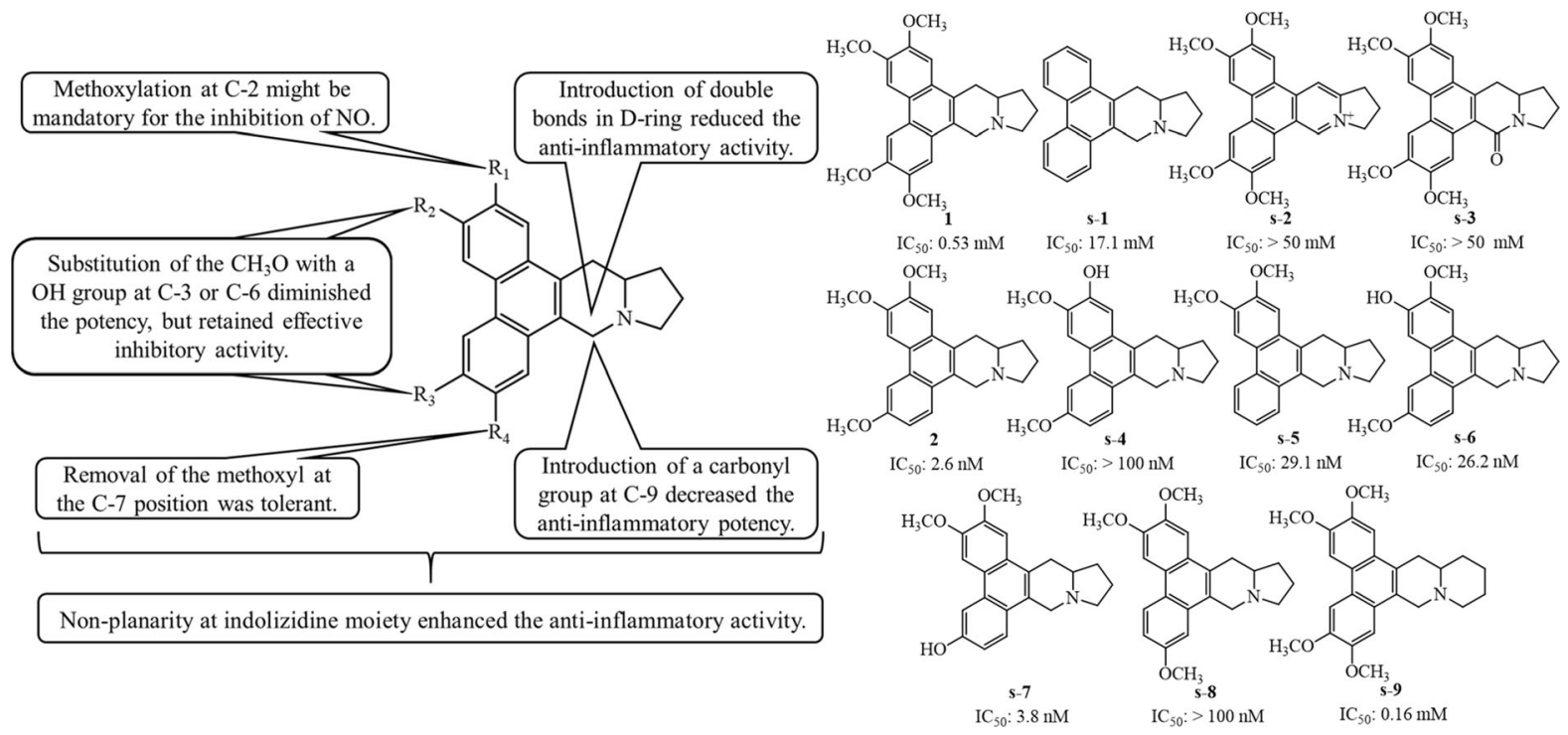

Fig. 10 Possible SARs of anti-inflammatory activity based on the NO suppression $\mathrm{IC}_{50}$ values of tylophorine (1) and antofine (2). Tylophorine (1) and its derivatives (s-1-s-3 and s-9) could

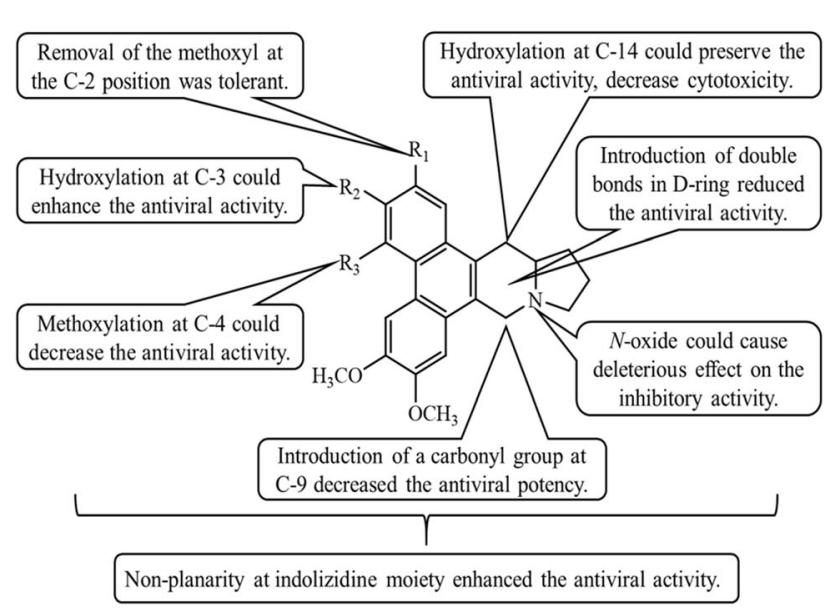

Fig. 11 Possible SARs of the anti-TGEV activity for PIA derivatives based on tylophorine (1). In the anti-TGEV assay, compounds $\mathbf{3 1}, \mathbf{3 3}$ and $\mathbf{3 7}$ were used as natural products from $T$.

function, which was confirmed by the NO suppression $\mathrm{IC}_{50}$ values of $\mathbf{s - 4}-\mathbf{s}-\mathbf{8}$ comparing with tylophorine (1) and antofine (2) (Min et al. 2010). Besides, keeping the non-planar structure of the indolizidine moiety was very vital for their anti-inflammatory activity. The quaternary PIA analogue, such as dehydrotylophorine $(\mathbf{s}-2)$, or the introduction of a keto group to C-9 (s-3), these structural modifications increased the rigidity be prepared by synthetic methods (Chuang et al. 2006). Antofine (2) and its synthesized derivatives $(\mathbf{s}-\mathbf{4}-\mathbf{S - 8})$ were obtained as described by $\mathrm{Fu}$ and his co-workers (Fu et al. 2007)

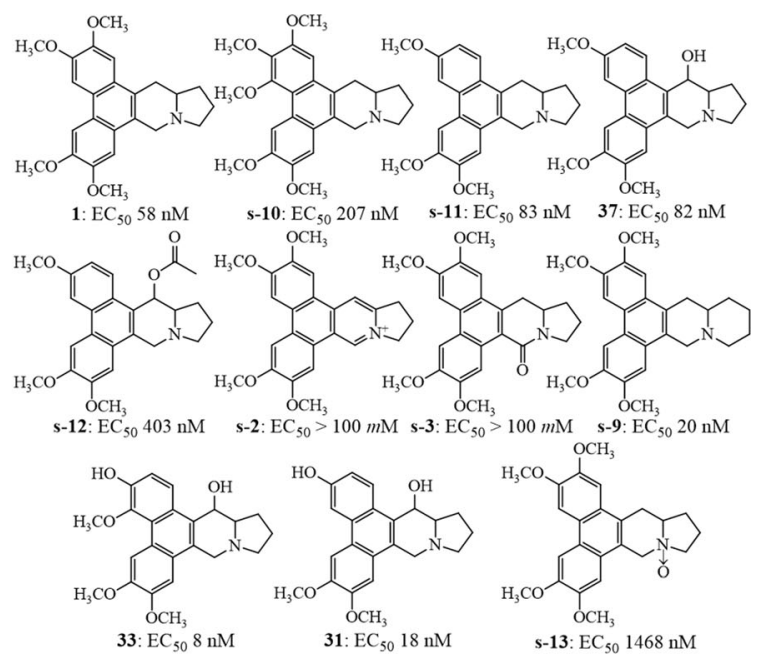

ovata (31 and 33) and T. indica (37). Other compounds were all synthesized derivatives (Yang et al. 2010)

and planarity of the whole structure skeleton or changed the amine group to an amide, could greatly reduce their bioeffects. Comparing the results of tylophorine (1) with 7-methoxycryptopleurine (s-9), it might deduce that the PQAs were more potent than their PIA counterparts for anti-inflammatory activity (Yang et al. 2007). 
<smiles>COc1cc2c3c(c4cc(OC)c(OC)cc4c2cc1OC)C(=O)N1CCC[C@H]1C3</smiles>

s-14, $48.1 \%$

$\mathbf{s - 1 5}, 15.3 \%$<smiles>CC=C(C)OC</smiles><smiles>CCc1c(CC)c2cc(OC)c(OC)cc2c2cc(OC)c(OC)cc12</smiles>

21, $54.1 \%$<smiles>COc1cc2c3c(c4cc(OC)c(OC)cc4c2cc1OC)N1CCC[C@@H](C1)[C@@H]3N</smiles>

s-20, $12.9 \%$<smiles>CCCCCCOc1ccc2c3c(c4cc(OC)c(OC)cc4c2c1)[C@H](N)[C@H]1CCCN3C1</smiles>

s-21, $48.2 \%$<smiles>COc1ccc2c3c(c4cc(OC)c(OC)cc4c2c1)[C@@H](N)[C@H]1CCCN3C1</smiles>

s-22, $72.4 \%$<smiles>COc1ccc2c3c(c4cc(OC)c(OC)cc4c2c1)CN1CCC[C@]1(CO)C3</smiles>
s-23, $34.7 \%$

s-24, $75.1 \%$

s-25, $23.5 \%$

s-26, $85.3 \%$<smiles>COc1cc2c3c(c4cc(OC)c(OC)cc4c2cc1OC)CN1CCCC1C3</smiles><smiles>C#CC[N+]12CCC[C@@H]1Cc1c(c3cc(OC)c(OC)cc3c3cc(OC)c(OC)cc13)C2</smiles>

s-28, $80.5 \%$ 
4Fig. 12 Structures of some typical PIA derivatives with their inhibition against TMV in vitro. In the anti-TMV assay, all the compounds were used as synthesized derivatives on the base of $(R / S)$-tylophorine or $(R / S)$-antofine. Their anti-TMV activity were revealed by the inhibition ratio $(\%)$ at $500 \mu \mathrm{g} / \mathrm{mL}$. The inhibitions of ningnanmycin, a commercial antiviral agent as the positive control, were about $70 \%$ in different anti-TMV assays in vitro

\section{Antiviral activity}

\section{Anti-coronaviral activity}

Tylophorine analogues, including naturally occurring and synthetic PIAs and PQAs, as potent inhibitors of enteropathogenic coronavirus transmissible gastroenteritis virus (TGEV), human severe acute respiratory syndrome coronavirus (SARS $\mathrm{CoV}$ ), and mouse hepatitis virus (MHV), were investigated in vitro (Yang et al. 2010; Lee et al. 2012). Among them, compounds 31 and 33, two natural PIA analogues isolated from $T$. ovata, and a synthetic PQA derivative, 7-methoxycryptopleurine (s-9), potently inhibited TGEV replication with $\mathrm{EC}_{50}$ values at 18,8 and $20 \mathrm{nM}$, respectively, and also possessed high selectivity index (SI) values. Tylophorine (1), 7-methoxycryptopleurine (s-9), and tylophorinine (37), were found to have profound activity against SARS CoV with $\mathrm{EC}_{50}$ values at $18,<5$, and $<5 \mathrm{nM}$ determined by cytopathic assay under microscopic observation. Further detailed antiviral mechanism of tylophorinebased compounds at molecular level was investigated. They could exert potent anti-TGEV replication by directly targeting the viral RNA/ribonucleoprotein (RNP) complex for viral replication/synthesis, and also could inhibit the nuclear factor $\kappa \mathrm{B}$ activation associated with IKK-2_I $\kappa$ B $\alpha /$ p65 axis and JAK pathway mediated p65 phosphorylation (Yang et al. 2017). Moreover, tylophorine (1) also potently inhibited the cytopathic effect and antiviral protein expression in infected DBT cells induced by MHV at $<0.2 \mu \mathrm{M}$ (Lee et al. 2012).

Based on the antiviral results of those naturally occurring and synthetic PIA and PQA derivatives, positive or negative structural requirements were also revealed (Fig. 11). Firstly, similar to that of antiinflammatory activity, the non-planar structure of the indolizidine moiety was also vital for their anti-TGEV activity, because of the great decrease bioeffects of $\mathbf{s - 2}$ and $\mathbf{s - 3}$ comparing to that of tylophorine (1). Secondly, hydroxylation at C-14 and C-3 contributed significantly to the inhibitory activity (31 to $\mathbf{3 7}$ ). However, introduction of an -OAc group into the $\mathrm{C}-14$ position decreased the activity (s-12 to 37). Moreover, introduction of a methoxyl group to position C-4 of tylophorine could decrease the inhibitory activity of the parent compound (s-10 to $\mathbf{1}$ ). Third, oxidation to the reactive nitrogen to form an $\mathrm{N}$-oxide also could decrease the inhibition, such as the great less potency of $\mathbf{s - 1 3}$ to that of $\mathbf{1}$. Besides, the PQAs deferred to the above conclusions generally and were more potent than their PIA counterparts for the antiviral activity (s9 to $\mathbf{1}$ ).

\section{Anti-HIV activity}

Interestingly, in an in vitro against human immunodeficiency virus (HIV) assay, dehydroantofine (69), but not $(R)$-antofine (2) isolated from $C$. chinensis, suppressed HIV-infected $\mathrm{H} 9$ cell growth significantly with an $\mathrm{EC}_{50}$ of $1.88 \mu \mathrm{g} / \mathrm{mL}$. This promising finding was the first example of a PIA analogue exhibiting anti-HIV activity. Thus, this compound type might provide a useful lead for anti-AIDS drug development (Wu et al. 2012).

Inhibitory activity against tobacco mosaic virus (TMV)

Plant viruses are pathogenic to more than 700 plants and cause significant yield losses in crop production worldwide. Among them, TMV has been voted as the most important plant virus in molecular plant pathology based on their perceived importance, scientifically or economically (Scholthof et al. 2011). For naturally occurring PIA analogues, $(R)$-antofine (2) was firstly found to have good antiviral activity against TMV in vitro (An et al. 2001). Subsequently, the other analogues, (R)-tylophorine (1), 6-O-desmethylantofine (14), and deoxytylophorinine (25), revealed nearly the same in vitro TMV assembly inhibition as (R)-antofine (2) (Huang et al. 2007; Wang et al. 2007). The possible molecular mechanism against TMV was speculated as that they likely exerted its virus inhibition by binding to oriRNA and interfering with virus assembly initiation (Xi et al. 2006). They also could bind selectively with RNA bulged structures to disrupt interaction between TMV RNA and coat protein (Gao 
et al. 2012). An enlightened antiviral assay was then applied to some PQA analogues, such as $(R)$-cryptopleurine $(\mathbf{8 1}),(R)$-boehmeriasin A $(\mathbf{8 2})$, and boehmeriasin $\mathrm{B}(\mathbf{8 5})$, they also displayed potent antiviral activity against TMV (Wang et al. 2012c).

Now, ningnanmycin and ribavirin are two kinds of successful registered anti-plant viral agents and widely used for the treatment of TMV infection. Interestingly, the above natural PIA and PQA analogues all exhibited higher inhibitory activity against TMV than ribavirin, $(R)$-antofine $(2), \quad(R)-6-\mathrm{O}-$ desmethylantofine (14), $(R)$-cryptopleurine $(\mathbf{8 1})$, and boehmeriasin B (85) even revealed the better antiviral activity in vitro and in vivo than that of ningnanmycin (Wu et al. 2013; Wang et al. 2012c). Due to their potent antiviral activity against TMV, $(R / S)$-antofine $(R / S)$-tylophorine, $(R / S)$-cryptopleurine and $(R / S)$ boehmeriasin A were abundantly used to present interesting targets for total or semi synthesis, and SARs study for the development of agrochemicals against TMV infection. Series of synthetic PIA and PQA derivatives along with natural analogues were systematically evaluated on their antiviral activity, in order to investigate the precise structural requirements for the inhibition of TMV (Fig. 12). Generally, PQA and PIA derivatives possessed the similar SARs for their antiviral activity against TMV infection (Wang et al. 2012c, 2014). More detailed SARs of PIAs were established as follows:

(1) The non-planar indolizidine moiety was the basic structural requirement for the anti-TMV effect.

Compound s-14, a synthetic derivative with a carbonyl group at C-9 of (S)-tylophorine (21), decreased the inhibitory activity in vitro. Its further dehydro-derivative in ring $\mathrm{E}, \mathbf{s - 1 5}$, almost lost its antiviral activity. These structural modifications all increased the rigidity and planarity of the whole structure skeleton (Wang et al. 2012b).

(2) The phenanthrene unit with hydroxy and alkoxy substituents were mandatory for the anti-TMV activity.

It seemed that 2,3,6- or 3,6,7trimethoxylphenanthrene unit was more excellent than 2,3,6,7-trtramethoxylphenanthrene unit for the antiviral activity, because both $( \pm)$-antofine $\quad(2,3,6$-trimethoxylphenanthrene unit) and ( $( \pm)$-deoxytylophorine (3,6,7trimethoxylphenanthrene unit) exhibited better anti-TMV effects than $( \pm)$-tylophorine (2,3,6,7trtramethoxylphenanthrene unit) (Wang et al. 2010, 2012b). Furthermore, for ( $R$ )-antofine (2), if the methoxyl at C-6 was changed to a hydroxyl group (14) or other hydrogen donor or electron-withdrawing groups (s-16-s-19), it was favorable for antiviral activity. Thus, antofine analogues with different C-6 substituents could be considered as a new class of antiviral agents (Wu et al. 2013).

(3) The polar substituents (amino or hydroxyl group) at C-14 could influence the bioeffects, however, relating to the phenanthrene substitution pattern.

As anti-TMV agents, introduction of a hydroxyl at C-14 position of $( \pm$ )-tylophorine could preserve the in vitro and in vivo antiviral activity, however, this structural modification could decrease the bioactivity of $( \pm)$-antofine and ( \pm )-deoxytylophorine (Wang et al. 2012b). The similar decreased tendency could be found for the 14- $\mathrm{NH}_{2}$ substituted $(S)$-tylophorine (s-20) and $(S)$-antofine (s-21), although the 14-aminodeoxytylophorinine (s-22) preserved the similar high antiviral activity as its parent molecule, deoxytylophorinine (25). However, with the further acetylation, 14-acetamino substituted $(S)$-tylophorine (s-23) and $(S)$-antofine (s-24) increased their bioeffects, while the anti-TMV activity of 14-acetamino substituted $(S)$ deoxytylophorinine (s-25) was decreased obviously (Wang et al. 2012a, b).

(4) Stereochemistry of C-13a with its substituted groups could change the bioactivity of PIAs.

Previously, Chemler summarized that C-2 methoxylation in the $R$-series, while C-7 methoxylation in the $S$-series, was very important for some PIAs to displayed their high anticancer activity, such as tylophorine, antofine, tylocrebrine and isotylocrebrine (Chemler 2009). Recent various antiviral bioassay against TMV basically approved this conclusion, especially confirmed by in vitro anti-TMV results of deoxytylophorine (Wang et al. 2012a) and 6-Odesmethylantofine (Wu et al. 2013). However, in vitro anti-TMV activity of $(R / S)$-tylophorine (Wang et al. 2010) and ( $R / S)$-antofine (Wang 
et al. 2012a; Su et al. 2016) was nearly equal. Furthermore, introduction of other groups, such as methyl, methylcarboxyl, and hydroxymethyl, at $\mathrm{C}$-13a of $(R / S)$-antofine, only the $\alpha$-hydroxymethyl substituted derivative (s-26) was evaluated to greatly enhance the anti-TMV effects (Su et al. 2014, 2016). These results obviously demonstrated that the spatial configuration of the molecules was crucial for keeping high bioactivities and introduction of suitable functional groups at the $\mathrm{C}-13 \mathrm{a}$ position could accelerate this trend.

(5) Salinization of N-10 could alter the anti-TMV activity because of the increasing stability and water solubility.

In general, PIAs salt derivates produced directly from ( \pm )-tylophorine with inorganic acids or organic acids could enhance their antiviral effects, only its carbazotic acid salt (s-27) decreased its bioactivity (Wang et al. 2010). However, introduction of different substituents at $\mathrm{N}-10$ to yield chiral quaternary ammonium salts could result in a negative effect for their anti-TMV activity, although the $(S)$-tylophorine derivative (s-28) produced with 3-bromoprop-1yne greatly enhanced the antiviral activity. Moreover, anti-TMV activity of these chiral quaternary ammonium salts varied significantly depending upon the chirality of C-13a and N-10 following this sequence: $13 \mathrm{a} S>13 \mathrm{a} R$, and trans $>$ cis (Han et al. 2018).

\section{Antifungal activity}

Recently, (R)-antofine (2) was investigated the inhibitory effect against plant-pathogenic fungi of Penicillium species. It showed strong antifungal activity against $P$. digitatum and $P$. italicum with an observed in vitro minimum inhibitory concentration (MIC, 1.56 and $1.56 \mu \mathrm{g} / \mathrm{mL}$ ) and a minimum fungicidal concentration (MFC, 12.5 and $6.25 \mu \mathrm{g} / \mathrm{mL}$ ). In vivo assays also showed that antofine could significantly reduce the incidence of green mold in citrus fruit. Both of them can be attributed to the disruption of the cell membrane integrity and energy metabolism (Xin et al. 2019; Chen et al. 2019b). Antifungal results indicated some PIAs could be used as natural fungicides for controlling postharvest green mold, blue mold, and sour rot disease of fruits and vegetables caused by Penicillium pathogens.

Insecticidal activity

Three PIA analogues, $(R)$-tylophorine $(\mathbf{1}),(R)$-antofine (2) and $(R)$-antofine $N$-oxide (41), were isolated from $C$. mongolicum based on the bioassay-guided tracing. They all showed toxic effect against Spodoptera litura and the aphid Lipaphis erysimi. Compound $\mathbf{4 1}$ was more toxic than $\mathbf{1}$ and $\mathbf{2}$, but they were all less active against $S$. litura than total alkaloids. The contact toxicity of these alkaloids against the aphid L. erysimi was significant, as the $24 \mathrm{~h}-\mathrm{LC}_{50}$ values of them and total alkaloids were 292.48, 367.21, 487.791 and $163.52 \mathrm{mg} / \mathrm{L}$, respectively. In the development disruption of $S$. litura larvae, the pupation and emergence rates decreased and the acute mortality increased significantly by day 3 after being injected in their body cavity with $10-40 \mathrm{mg} / \mathrm{L}$ of total alkaloid. The ecdysone titer of treated $S$. litura larvae and prepupae also declined with increasing alkaloid concentration. The alkaloids of $C$. mongolicum could be used as potential insect growth inhibitors (Ge et al. 2015).

\section{Anti-angiogenic activity}

Both $(R)$-tylophorine $(\mathbf{1})$ and $(R)$-antofine (2) were selected to the anti-angiogenic assay based on their excellent anti-proliferative activities in a variety of cancer cells. Antofine (2) could effectively inhibit the proliferation of both endothelial cell types, with $\mathrm{IC}_{50}$ $435.5 \mathrm{nM}$ in cultured mouse embryonic stem/embryoid body-derived endothelial cell and $36.0 \mathrm{nM}$ in vascular endothelial growth factor (VEGF)-induced human umbilical vein endothelial cells (HUVECs), respectively. The underlying mechanism of its antiangiogenic activity was, in part, associated with the modulation of AKT/mTOR and AMP-activated protein kinase (AMPK) signaling in VEGF-stimulated HUVECs. (Oh et al. 2017). Tylophorine (1) potently inhibited the PDGF-BB-induced proliferation of rat aortic vascular smooth muscle cells (VSMCs) in a concentration-dependent manner with an $\mathrm{IC}_{50}$ of $0.13 \mu \mathrm{M}$, by arresting cells in $\mathrm{G} 1$ phase of the cell cycle as well as migration in activated VSMCs. It could decrease de novo protein synthesis, result in downregulation of cyclin D1 and prevent neointimal thickening in human saphenous vein organ culture 
(Joa et al. 2019). Anti-angiogenic results indicated tylophorine (1) and antofine (2) could be used as promising lead compounds for further studies towards the treatment of vasculo-proliferative disorders, such as restenosis.

Anti-adipogenic activity

Antofine (1) was revealed with a novel anti-adipogenic activity at low concentrations (0.01-10 nM) to repress the proliferation and differentiation of preadipocyte 3T3-L1 cells without associated cytotoxicity. Its underlying molecular mechanism was to suppress the expression of endogenous peroxisome proliferator-activated receptor $\gamma(\operatorname{PPAR} \gamma)$ protein in adipocytes, leading to the attenuation of PPAR $\gamma$ induced adipogenic gene expression and consequent inhibition of adipocyte differentiation. Antofine could be used as a promising candidate in the control of obesity as well as tumor (Jang et al. 2014).

\section{Antiplasmodial activity}

Up to date, only phenanthroindolizine-type alkaloids in the extract of $F$. septica were reported on antimalarial activities. From the most potent chloroformsoluble portion, a new seco-phenanthroindolizinetype alkaloid, 4a,b-seco-dehydroantofine $(\mathbf{8 0})$ with tylophoridicine D (67), dehydrotylophorine (68), and dehydroantofine (69), were isolated with $\mathrm{IC}_{50}$ values against a chloroquine-sensitive strain (3D7) of Plasmodium falciparum at 4.0, 0.058, 0.42, and $0.028 \mu \mathrm{M}$, respectively. Moreover, both 67 and 69 showed potent antimalarial activities comparative to those of positive controls, artemisinin $\left(\mathrm{IC}_{50} 0.025 \mu \mathrm{M}\right)$ and chloroquine $\left(\mathrm{IC}_{50} 0.036 \mu \mathrm{M}\right)$, but were inactive against mouse fibroblast cells L929. These results suggested that PIAs might be acted as therapeutic leads for new antimalarial drugs (Kubo et al. 2016).

\section{Oviposition-stimulatory activity}

A common danaid butterfly, Ideopsis similis (L.), exclusively attacks an asclepiadaceous plant, $T$. tanakae. In its pupae and imagines, trace of PIAs was detected as the sequestration phenomenon (Komatsu et al. 2001; Abe et al. 2001). A total of 12 PIAs isolated from $T$. tanakae were selected to evaluate their oviposition-stimulatory activity at a dose of
$100 \mathrm{mg} / \mathrm{cm}^{2}$ on female danaid butterfly. Favorably, (+)-isotylocrebrine (27), (+)-3-demethylisotylocrebrine (26), (+)-isotylocrebrine $N$-oxide (61), and (-)7-demethyltylophorine (15), induced the females to lay 28, 23, 15 and 25 eggs, respectively, larger numbers than that of water as the control (not more than 7 eggs). However, female egg-laying was much higher in response to a mixture of those alkaloids, which strongly suggested that host selection by the butterfly was mediated by the synergistic action of several PIAs present in the host plant. For the SARs, it was apparent that the introduction of a hydroxy group at $\mathrm{C}-14$ and the formation of $\mathrm{N}$-oxide considerably depressed the activity. In those with $R$-configuration, the presence of a hydroxy group rather than a methoxy one at C-7 appeared to increase stimulation of oviposition (Honda et al. 2001).

\section{Application prospects of PIAs and PQAs}

Although tylocrebrine (12) was advanced to clinical trials but failed due to its central nervous system (CNS) toxicity manifested as disorientation and ataxia, naturally occurring PIA and PQA analogues along with their total or semi synthetic derivatives still attract great attention all along as potent anti-cancer agents because of their excellent biological activities, such as the isolation of new natural analogues (Yap et al. 2016; Al-Khdhairawi et al. 2017; Chen et al. 2019a; Thuy et al. 2019), new synthetic methods and new anti-cancer mechanism on active agents (Chen et al. 2016; Wang et al. 2018; Jo et al. 2019; Shimada et al. 2019), etc. Therefore, it is worthwhile to carry out further investigations on natural species and organic reactions for more novel PIA and PQA analogues with great bioeffects. To investigate the precise structural requirements of these kinds of compounds for suppressing their neurotoxicity and other toxic effects also may be one of the study directions.

In addition, PIA and PQA analogues probably can act as the candidate of new antiviral agents against coronavirus. Pneumonia outbreaks caused by novel coronavirus, such as the previous SARS and the present COVID-19 caused by SARS-CoV-2 in the begin of the year 2020, are great threat to the public health, however, with few effective therapeutic drugs. Some PIA and PQA analogues, such as 
tylophorine (1), tylophorinine (37), and 7-methoxycryptopleurine (s-9), were found to have high potency against SARS CoV and TGEV in vitro at low nanomolar level with high oral availability in rats, which suggested they could be used as potential therapeutic agents for coronavirus infections (Yang et al. 2010; Lee et al. 2012). Moreover, the novel coronavirus, SARS-CoV-2, has been revealed almost identical to each other and shared $79.5 \%$ sequence identify to SARS-CoV (Zhou et al. 2020), these PIA and PQA analogues may be effective to CoV-2019. Therefore, it is worthwhile to carry out further investigations on PIA and PQA analogues as potent antiviral agents.

In addition, application of some PIAs as antifungal and antiviral agents against plant-pathogenic fungi of Penicillium species and TMV indicated this kind of compounds can be used as potential agrochemicals. Naturally occurring autofine (2), deoxytylophorinine (11), and 6-O-desmethylantofine (14), along with

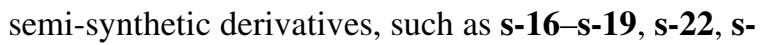
24, s-26 and s-28, etc., they all displayed the similar or more potent in vitro and in vivo antiviral activity than ningnanmycin, the successful registered anti-plant viral agents for the treatment of TMV infection. As the natural product based antiviral agents, some PIA and PQA derivatives can be used as ideal lead structures to develop more attractive agrochemicals with easy decomposition, environmental friendliness, specification to targeted species, or unique mode of action.

\section{Conclusions}

Besides of their promising anticancer activities with the structural modifications, this article mainly presented an overview of naturally occurring PIAs and PQAs obtained in the last two decades, focusing on their origins, structural variety, key spectroscopic characteristics, other biological activity with structure-activity relationships and application prospects. Their structural skeletons could suffer different modifications, such as oxidation (hydroxylation and carbonylation), dehydration, to result in the structural variety and associating with a wide spectrum of biological activities. Some PIA and PQA analogues exhibited very significant antiviral potentiality in nanomolar level against coronavirus. Moreover, their antifungal activity against Penicillium species and
anti-TMV applications revealed their potentiality used as attractive agrochemicals. In brief, structural variations with excellent biological activities suggested these two kinds of compounds might be worthy of further study and may be valuable for the development of new drug or agrochemical candidates.

Acknowledgements This work was financially supported by the Innovation Project of Shandong Academy of Medical Sciences (2019), the Industrial Upgrading Project of Shandong Agricultural Science and Technology Fund (2019YQ033).

\section{References}

Abe F, Iwase Y, Yamauchi T, Honda K, Omura H, Hayashi N (2001) Sequestration of phenanthroindolizidine alkaloids by an Asclepiadaceae feeding danaid butterfly, Ideopsis similis. Phytochemistry 56:697-701

Al-Khdhairawi AAQ, Krishnan P, Mai CW, Chung FF, Leong CO, Yong KT, Chong KW, Low YY, Kam TS, Lim KH (2017) A bis-benzopyrroloisoquinoline alkaloid incorporating a cyclobutane core and a chlorophenanthroindolizidine alkaloid with cytotoxic activity from Ficus fistulosa var. tengerensis. J Nat Prod 80:2734-2740

An TY, Huang RQ, Yang Z, Zhang DK, Li GR, Yao YC, Gao J (2001) Alkaloids from Cynanchum komarovii with inhibitory activity against the tobacco mosaic virus. Phytochemistry 58:1267-1269

Cai XF, Jin X, Lee D, Yang YT, Lee K, Hong YS, Lee JH, Lee JJ (2006) Phenanthroquinolizidine alkaloids from the roots of Boehmeria pannosa potently inhibit hypoxia-inducible factor-1 in AGS human gastric cancer cells. J Nat Prod 69:1095-1097

Chemler SR (2009) Phenanthroindolizidines and phenanthroquinolizidines: promising alkaloids for anti-cancer therapy. Curr Bioact Compd 5:2-19

Chen CY, Zhu GY, Wang JR, Jiang ZH (2016) Phenanthroindolizidine alkaloids from Tylophora atrofolliculata with hypoxia-inducible factor-1 (HIF-1) inhibitory activity. RSC Adv 6:79958-79967

Chen CY, Zhu GY, Xie TG, Jiang PC, Wang GR, Jiang ZH (2019a) A phenanthroindolizidine glycoside with HIF-1 inhibitory activity from Tylophora atrofolliculata. Phytochem Lett 31:39-42

Chen CY, Qi WW, Peng X, Chen JY, Wan CP (2019b) Inhibitory effect of 7-demethoxytylophorine on Penicillium italicum and its possible mechanism. Microorganisms 7:36

Chou ST, Jung F, Yang SH, Chou HL, Jow GM, Lin JC (2017) Antofine suppresses endotoxin-induced inflammation and metabolic disorder via AMP-activated protein kinase. Pharmacol Res Perspect 5:e00337

Chuang TH, Lee SJ, Yang CW, Wu PL (2006) Expedient synthesis and structure-activity relationships of phenanthroindolizidine and phenanthroquinolizidine alkaloids. Org Biomol Chem 4:860-867

Cui L, Abliz Z, Xia M, Zhao L, Gao S, He W, Xiang Y, Liang F, $\mathrm{Yu} S$ (2004) On-line identification of 
phenanthroindolizidine alkaloids in a crude extract from Tylophora atrofolliculata by liquid chromatography combined with tandem mass spectrometry. Rapid Commun Mass Spectrom 18:184-190

Damu AG, Kuo PC, Shi LS, Li CY, Kuoh CS, Wu PL, Wu TS (2005) Phenanthroindolizidine alkaloids from the stems of Ficus septica. J Nat Prod 68:1071-1075

Damu AG, Kuo PC, Shi LS, Li CY, Su CR, Wu TS (2009) Cytotoxic phenanthroindolizidine alkaloids from the roots of Ficus septica. Planta Med 75:1152-1156

de Fatima PM, Rochaisa C, Patrick Dallemagnea P (2015) Recent advances in phenanthroindolizidine and phenanthroquinolizidine derivatives with anticancer activities. Anti-Cancer Agent Med 15:1080-1091

Dhiman M, Khanna A, Manj SL (2013) A new phenanthroindolizidine alkaloid from Tylophora indica. Chem Pap 67:245-248

Fu Y, Lee SK, Min HY, Lee T, Lee J, Cheng M, Kim S (2007) Synthesis and structure-activity studies of antofine analogues as potential anticancer agents. Bioorg Med Chem Lett 17:97-100

Gao S, Zhang RY, Yu ZH, Xi Z (2012) Antofine analogues can inhibit tobacco mosaic virus assembly through smallmolecule-RNA interactions. Chem Bio Chem 13:1622-1627

Ge Y, Liu PP, Yang R, Zhang L, Chen HX, Camara I, Liu YQ, Shi WP (2015) Insecticidal constituents and activity of alkaloids from Cynanchum mongolicum. Molecules 20:17483-17492

Gellert E (1982) The indolizidine alkaloids. J Nat Prod 45:50-73

Govindachari TR, Viswanathan N (1978) Recent progress in the chemistry of phenanthroindolizidine alkaloids. Heterocycles 11:587-613

Han GF, Chen LW, Wang Q, Wu M, Liu YX, Wang QM (2018) Design, synthesis, and antitobacco mosaic virus activity of water soluble chiral quaternary ammonium salts of phenanthroindolizidines alkaloids. J Agric Food Chem 66:780-788

Hoffmann JJ, Luzbetak DJ, Torrance SJ, Cole JR (1978) Cryptopleurine, cytotoxic agent from Boehmeria caudata (Urticaceae) and Cryptocarya laevigata (Lauraceae). Phytochemistry 17:1448

Honda K, Omura H, Hayashi N, Abe F, Yamauchi T (2001) Oviposition-stimulatory activity of phenanthroindolizidine alkaloids of host-plant origin to a danaid butterfly, Ideopsis similis. Physiol Entomol 26:6-10

Huang X, Gao S, Fan L, Yu S, Liang X (2004) Cytotoxic alkaloids from the roots of Tylophora atrofolliculata. Planta Med 70:441-445

Huang ZQ, Liu YX, Fan ZJ, Wang QM, Li GR, Yao YC, Yu XS, Huang RQ (2007) Antiviral activity of alkaloids from Cynanchum komarovii. Fine Chem Intermed 37(20-24):46

Jang EJ, Kim HK, Jeong H, Lee YS, Jeong MG, Bae SJ, Kim S, Lee SK, Hwang ES (2014) Anti-adipogenic activity of the naturally occurring phenanthroindolizidine alkaloid antofine via direct suppression of PPARg expression. Chem Biodivers 11:962-969

Jo YI, Burke MD, Cheon CH (2019) Modular syntheses of phenanthroindolizidine natural products. Org Lett $21: 4201-4204$
Joa H, Blažević T, Grojer C, Zeller I, Heiss EH, Atanasov AG, Feldler I, Gruzdaitis P, Czaloun C, Proksch P, Messner B, Bernhard D, Dirsch VM (2019) Tylophorine reduces protein biosynthesis and rapidly decreases cyclin D1, inhibiting vascular smooth muscle cell proliferation in vitro and in organ culture. Phytomedicine 60:152938

Johns SR, Lamberton JA, Sioumis AA, Willing RI (1970) New alkaloids from Cryptocarya pleurosperma (Lauraceae): the structures of cryptopleuridine and cryptopleurospermine. Aust J Chem 23:353-361

Karnick CR (1975) Phytochemical investigations of some Tylophora species found in India. Planta Med 27:333-336

Komatsu H, Watanabe M, Ohyama M, Enya T, Koyama K, Kanazawa T, Kawahara N, Sugimura T, Wakabayashi K (2001) Phenanthroindolizidine alkaloids as cytotoxic substances in a danaid butterfly, Ideopsis similis, against human cancer cells. J Med Chem 44:1833-1836

Krmpotic E, Farnsworth NR, Messmer WM (1972) Cryptopleurine, an active antiviral alkaloid from Boehmeria cylindrica (L.) Sw. (Urticaceae). J Pharm Sci 61:1508-1509

Kubo M, Yatsuzuka W, Matsushima S, Harada K, Inoue Y, Miyamoto H, Matsumoto M, Fukuyama Y (2016) Antimalarial phenanthroindolizine alkaloids from Ficus septica. Chem Pharm Bull 64:957-960

Lande DE (1948) The alkaloids of Cryptocarya pleurosperma. Aust J Exp Biol Med Sci 34:181-187

Lee YZ, Huang CW, Yang CW, Hsu HY, Kang IJ, Chao YS, Chen IS, Chang HY, Lee SJ (2011) Isolation and biological activities of phenanthroindolizidine and septicine alkaloids from the Formosan Tylophora ovata. Planta Med 77:1932-1938

Lee YZ, Yang CW, Hsu HY, Qiu YQ, Yeh TK, Chang HY, Chao YS, Lee SJ (2012) Synthesis and biological evaluation of tylophorine-derived dibenzoquinolines as orally active agents: exploration of the role of tylophorine $\mathrm{E}$ ring on biological activity. J Med Chem 55:10363-10377

Li Z, Jin Z, Huang R (2001) Isolation, total synthesis and biological activity of phenanthroindolizidine and phenanthroquinolizidine alkaloids. Synthesis 16:2365-2378

Luo Y, Liu Y, Luo D, Gao X, Li B, Zhang G (2003) Cytotoxic alkaloids from Boehmeria siamensis. Planta Med 69:842-845

Min HY, Song SH, Lee B, Kim S, Lee SK (2010) Inhibition of lipopolysaccharide-induced nitric oxide production by antofine and its analogues in RAW 264.7 macrophage cells. Chem Biodivers 7:409-414

Nagle DG, Zhou YD (2006) Natural product-based inhibitors of hypoxia-inducible factor-1 (HIF-1). Curr Drug Targets 7:355-369

Nakano D, Ishitsuka K, Ikeda M, Tsuchihashi R, Okawa M, Okabe H, Tamura K, Kinjo J (2015) Screening of promising chemotherapeutic candidates from plants against human adult T-cell leukemia/lymphoma (IV): phenanthroindolizidine alkaloids from Tylophora tanakae leaves. J Nat Med. https://doi.org/10.1007/s11418-0150906-8

Oh J, Kim GD, Kim S, Lee SK (2017) Antofine, a natural phenanthroindolizidine alkaloid, suppresses angiogenesis via regulation of $\mathrm{AKT} / \mathrm{mTOR}$ and AMPK pathway in endothelial cells and endothelial progenitor cells derived 
from mouse embryonic stem cells. Food Chem Toxicol 107:201-207

Othman WNNW, Sivasothy Y, Liew SY, Mohamad J, Nafiah MA, Ahmad K, Litaudon M, Awanga K (2017) Alkaloids from Cryptocarya densiflora Blume (Lauraceae) and their cholinesterase inhibitory activity. Phytochem Lett 21:230-236

Rathnagiriswaran AN, Venkatachalam K (1935) The chemical examination of tylophora asthmatica and isolation of the alkaloids tylophorine and tylophorinine. Indian J Med Res 22:433

Saifah E, Kelley CJ, Leary JD (1983) Constituents of the leaves of Cissus rheifolia. J Nat Prod 46:353-358

Scholthof KB, Adkins S, Czosnek H, Palukaitis P, Jacquot E, Hohn T, Hohn B, Saunders K, Candresse T, Ahlquist P, Hemenway C, Foster GD (2011) Top 10 plant viruses in molecular plant pathology. Mol Plant Pathol 12:938-954

Shimada K, Suzuki M, Yahaba K, Aoyagi S, Takikawa Y, Korenaga T (2019) An efficient synthesis of phenanthroindolizidine core via hetero Diels-Alder reaction of in situ generated $\alpha$-allenylchalcogenoketenes with cyclic imines. Nat Prod Commun 7:1-13

Song J, Kwon Y, Kim S et al (2015) Antitumor activity of phenanthroindolizidine alkaloids is associated with negative regulation of met endosomal signaling in renal cancer cells. Chem Biol 22:1-12

Stærk D, Lykkeberg AK, Christensen J, Budnik BA, Abe F, Jaroszewski JW (2002) In vitro cytotoxic activity of phenanthroindolizidine alkaloids from Cynanchum vincetoxicum and Tylophora tanakae against drug-sensitive and multidrug-resistant cancer cells. J Nat Prod 65:1299-1302

Stærk D, Nezhad KB, Asili J, Emami SA, Ahi A, Sairafianpour M, Jaroszewski JW (2005) Phenanthroindolizidine alkaloids from Vincetoxicum pumilum. Biochem Syst Ecol 33:957-960

Stoye A, Peez TE, Opatz T (2013) Left, right, or both? On the configuration of the phenanthroindolizidine alkaloid tylophorine from Tylophora indica. J Nat Prod 76:275-278

Su B, Cai CL, Deng M, Liang DM, Wang LZ, Wang QM (2014) Design, synthesis, antiviral activity, and SARs of 13asubstituted phenanthroindolizidine alkaloid derivatives. Bioorg Med Chem Lett 24:2881-2884

Su B, Cai CL, Deng M, Liang DM, Wang QM (2016) Spatial configuration and three-dimensional conformation directed design, synthesis, antiviral activity, and structure-activity relationships of phenanthroindolizidine analogues. J Agric Food Chem 64:2039-2045

Subramaniam G, Ang KKH, Ng S, Buss AD, Mark S, Butler MS (2009) A benzopyrroloisoquinoline alkaloid from Ficus fistulosa. Phytochem Lett 2:88-90

Thuy ADT, Thanh VTT, Mai HDT, Le HT, Litaudon M, Nguyen VH, Chau VM, Pham VC (2019) Cytotoxic alkaloids from leaves of Pilea aff. Martini. Planta Med 85:496-502

Tian Y, He J, Zhan R, Lv H, Ma S, Chen Y, Yu S, Chen X, Wu $\mathrm{Y}, \mathrm{He} \mathrm{W}$, Abliz Z (2012) Integrated rapid resolution liquid chromatography-tandem mass spectrometric approach for screening and identification of metabolites of the potential anticancer agent 3,6,7-trimethoxyphenanthroindolizidine in rat urine. Anal Chim Acta 731:60-67
Wang QM, Yao YC, Huang RQ, Fan ZJ, Li GR, Yu XS (2007) Antiviral activity of antofine from Cynanchum komarovii. Agrochemicals 46:425-427

Wang K, Su B, Wang Z, Wu M, Li Z, Hu Y, Fan Z, Mi N, Wang Q (2010) Synthesis and antiviral activities of phenanthroindolizidine alkaloids and their derivatives. J Agric Food Chem 58:2703-2709

Wang Z, Wang L, Ma S, Liu Y, Wang L, Wang Q (2012a) Design, synthesis, antiviral activity, and SARs of 14-aminophenanthroindolizidines. J Agric Food Chem 60:5825-5831

Wang ZW, Wei P, Wang LZ, Wang QM (2012b) Design, synthesis, and anti-tobacco mosaic virus (TMV) activity of phenanthroindolizidines and their analogues. J Agric Food Chem 60:10212-10219

Wang Z, Feng A, Cui M, Liu Y, Wang L, Wang Q (2012c) First discovery and stucture-activity relationship study of phenanthroquinolizidines as novel antiviral agents against tobacco mosaic virus (TMV). PLoS One 7:e52933

Wang Z, Feng A, Cui M, Liu Y, Wang L, Wang Q (2014) Design, synthesis, anti-tobacco mosaic virus (TMV) activity, and SARs of 7-methoxycryptopleurine derivatives. Chem Biol Drug Des 84(5):531-542

Wang RB, Lv HN, Zhu SS, Ren XD, Xu S, Ma SG, Liu YB, Qu J, Yu SS (2018) A novel and practical synthesis of CAT3: a phenanthroindolizidine alkaloid with potential in treating glioblastoma. RSC Adv 8:29301-29308

Wu PL, Rao KV, Su CH, Kuoh CS, Wu TS (2002) Phenanthroindolizidine alkaloids and their cytotoxicity from the leaves of Ficus septica. Heterocycles 57:2401-2408

Wu TS, Su CR, Lee KH (2012) Cytotoxic and anti-HIV phenanthroindolizidine alkaloids from Cryptocarya chinensis. Nat Prod Commun 7:725-727

Wu M, Han GF, Wang ZW, Liu YX, Wang QM (2013) Synthesis and antiviral activities of antofine analogues with different C-6 substituent groups. J Agric Food Chem 61:1030-1035

Xi Z, Zhang RY, Yu ZH, Ouyang D (2006) The interaction between tylophorine B and TMV RNA. Bioorg Med Chem Lett 16:4300-4304

Xiang Y, Abliz Z, Li LJ, Huang XS, Yu SS (2002) Study of structural characteristic features of phenanthroindolizidine alkaloids by fast atom bombardment with tandem mass spectrometry. Rapid Commun Mass Spectrom 16:1668-1674

Xin ZT, OuYang QL, Wan CP, Che JX, Li L, Chen JY, Tao NG (2019) Isolation of antofine from Cynanchum atratum Bunge (Asclepiadaceae) and its antifungal activity against Penicillium digitatum. Postharvest Biol Technol 157:110961

Yang CW, Chen WL, Wu PL, Tseng HY, Lee SJ (2006) Antiinflammatory mechanisms of phenanthroindolizidine alkaloids. Mol Pharmacol 69:749-758

Yang CW, Chuang TH, Wu PL, Huang WH, Lee SJ (2007) Antiinflammatory effects of 7-methoxycryptopleurine and structure-activity relations of phenanthroindolizidines and phenanthroquinolizidines. Biochem Biophys Res Commun 354:942-948

Yang CW, Lee YZ, Kang IJ, Barnard DL, Jan JT, Lin D, Huang CW, Yeh TK, Chao YS, Lee SJ (2010) Identification of phenanthroindolizines and phenanthroquinolizidines as 
novel potent anti-coronaviral agents for porcine enteropathogenic coronavirus transmissible gastroenteritis virus and human severe acute respiratory syndrome coronavirus. Antivir Res 88:160-168

Yang CW, Lee YZ, Hsu HY, Shih C, Chao YS, Chang HY, Lee SJ (2017) Targeting coronaviral replication and cellular JAK2 mediated dominant NF- $\kappa \mathrm{B}$ activation for comprehensive and ultimate inhibition of coronaviral activity. Sci Rep 7:4105

Yap VA, Loong BJ, Ting KN, Loh SH, Yong KT, Low YY, Kam TS, Lim KH (2015) Hispidacine, an unusual 8,4-oxyneolignan-alkaloid with vasorelaxant activity, and hispiloscine, an antiproliferative phenanthroindolizidine alkaloid, from Ficus hispida Linn. Phytochemistry 109:96-102

Yap VA, Qazzaz ME, Raja VJ, Bradshaw TD, Loh HS, Sim KS, Yong KT, Low YY, Lim KH (2016) Fistulopsines A and B antiproliferative septicine-type alkaloids from Ficus fistulosa. Phytochem Lett 15:136-141
Yu F, Lv H, Dong W, Ye J, Hao H, Ma S, Yu S, Liu Y (2015) Development and validation of a liquid chromatographytandem mass spectroscopy method for simultaneous determination of (+)-(13aS)-deoxytylophorinine and its pharmacologically active 3-O-desmethyl metabolite in rat plasma. J Pharm Biomed 107:223-228

Zhou P, Yang XL, Wang XG, Hu B, Zhang L, Zhang W, Si HR, Zhu Y, Li B, Huang CL, Chen HD, Chen J, Luo Y, Guo H, Jiang RD, Liu MQ, Chen Y, Shen XR, Wang X, Zheng XS, Zhao K, Chen QJ, Deng F, Liu LL, Yan B, Zhan FX, Wang YY, XiaoGF SZ (2020) Discovery of a novel coronavirus associated with the recent pneumonia outbreak in humans and its potential bat origin. BioRxiv. https://doi.org/10. 1101/2020.01.22.914952

Publisher's Note Springer Nature remains neutral with regard to jurisdictional claims in published maps and institutional affiliations. 